\title{
Workplace neighbourhood built environment and workers' physically-active and sedentary behaviour: a systematic review of observational studies
}

Chien-Yu Lin ${ }^{1 *}$ (D), Mohammad Javad Koohsari ${ }^{2,3,4}$, Yung Liao ${ }^{2,5}$, Kaori Ishii ${ }^{2}$, Ai Shibata ${ }^{6}$, Tomoki Nakaya ${ }^{7}$, Gavin R. McCormack ${ }^{2,8}$, Nyssa Hadgraft ${ }^{9}$, Neville Owen ${ }^{4,9}$ and Koichiro Oka ${ }^{2}$

\begin{abstract}
Background: Many desk-based workers can spend more than half of their working hours sitting, with low levels of physical activity. Workplace neighbourhood built environment may influence workers' physical activities and sedentary behaviours on workdays. We reviewed and synthesised evidence from observational studies on associations of workplace neighbourhood attributes with domain-specific physical activity and sedentary behaviour and suggested research priorities for improving the quality of future relevant studies.

Methods: Published studies were obtained from nine databases (PubMed, Web of Science, PsycINFO, Scopus, Transport Research International Documentation, MEDLINE, Cochrane, Embase, and CINAHL) and crosschecked by Google Scholar. Observational studies with quantitative analyses estimating associations between workplace neighbourhood built environment attributes and workers' physical activity or sedentary behaviour were included. Studies were restricted to those published in English language peer-reviewed journals from 2000 to 2019.

Results: A total of 55 studies and 455 instances of estimated associations were included. Most instances of potential associations of workplace neighbourhood built environment attributes with total or domain-specific (occupational, transport, and recreational) physical activity were non-significant. However, destination-related attributes (i.e., longer distances from workplace to home and access to car parking) were positively associated with transport-related sedentary behaviour (i.e., car driving).

Conclusions: The findings reinforce the case for urban design policies on designing mixed-use neighbourhoods where there are opportunities to live closer to workplaces and have access to a higher density of shops, services, and recreational facilities. Studies strengthening correspondence between the neighbourhood built environment attributes and behaviours are needed to identify and clarify potential relationships.
\end{abstract}

Protocol registration: The protocol of this systematic review was registered on the International Prospective Register of Systematic Reviews (PROSPERO) on 2 December 2019 (registration number: CRD42019137341).

Keywords: Worksite, Employee, Walkability, Physical activity, Sitting

\footnotetext{
* Correspondence: chienyulin@akane.waseda.jp

'Graduate School of Sport Sciences, Waseda University, 2-579-15 Mikajima,

Tokorozawa, Saitama 359-1192, Japan

Full list of author information is available at the end of the article
}

\section{$\triangle B M C$}

(c) The Author(s). 2020 Open Access This article is licensed under a Creative Commons Attribution 4.0 International License, which permits use, sharing, adaptation, distribution and reproduction in any medium or format, as long as you give appropriate credit to the original author(s) and the source, provide a link to the Creative Commons licence, and indicate if changes were made. The images or other third party material in this article are included in the article's Creative Commons licence, unless indicated otherwise in a credit line to the material. If material is not included in the article's Creative Commons licence and your intended use is not permitted by statutory regulation or exceeds the permitted use, you will need to obtain permission directly from the copyright holder. To view a copy of this licence, visit http://creativecommons.org/licenses/by/4.0/ The Creative Commons Public Domain Dedication waiver (http://creativecommons.org/publicdomain/zero/1.0/) applies to the data made available in this article, unless otherwise stated in a credit line to the data. 


\section{Background}

Many desk-based workers spend the majority of their working hours being sedentary; this is markedly greater than the proportion of time spent sedentary during nonworking hours $[1,2]$. An ecological model of health behaviour suggests that workers' physically-active and sedentary behaviours are influenced by multiple factors [3]. Among the multiple influences, built environment factors can be particularly influential on desk-based workers' sedentary behaviour [4]. The workplace built environment comprises the built environment attributes inside (e.g., workstations and spatial layout of buildings) and on the land parcel of the workplace building (e.g., workplaceexclusive car parking) and the neighbourhood surrounding the workplace (e.g., neighbourhood walkability and destination access). Previous reviews relating to the workplace built environment have mainly focused on examining inside environments as potential influences on physically-active and sedentary behaviours during work time [4-7]. However, workplace neighbourhood environment may also be an important influence on the physical activity and sedentary behaviours of workers. Given there is greater capacity for workers to undertake moderate-to vigorous-intensity physical activity (e.g., walking and cycling) in the neighbourhood around and beyond the immediate workplace setting [8], the influence of workplace neighbourhood environment merits examination [9].

Synthesising the broader body of empirical evidence relating to workplace neighbourhood built environment is essential for informing urban design policies to support physical activity and reduce sedentary behaviours among workers. A previous systematic review examining associations of workplace built environments, both inside and neighbourhood attributes, with physical activity and sedentary behaviour reported inconclusive findings for neighbourhood attributes [10]. However, the search strategy used did not employ specific terms relating to characteristics of the neighbourhood built environment such as neighbourhood walkability, destinations, and safety. Furthermore, the previous findings did not distinguish the purpose of active and sedentary behaviours (e.g., for occupation, transport, or recreation). Therefore, the associations may be confounded as the environmental correlates varied by domains $[11,12]$.

Therefore, the aim of our review was to examine findings from observational studies and synthesise current evidence on associations of workplace neighbourhood built environment attributes (including those not located on the workplace precinct), with domain-specific physical activity and sedentary behaviour among desk-based workers. We further provided suggestions based on the results for improving the evidence on urban design policies to influence workers' physical activity and sedentary behaviours.

\section{Methods}

The protocol of this systematic review was published on the International Prospective Register of Systematic Reviews (PROSPERO) on 2 December 2019 (registration number: CRD42019137341).

\section{Database search strategy}

This systematic review was conducted in October 2019 following the Preferred Reporting Items for Systematic Reviews and Meta-Analyses (PRISMA) guidelines [13]. Systematic searches were conducted in nine databases: PubMed, Web of Science, PsycINFO, Scopus, Transport Research International Documentation, MEDLINE, Cochrane, Embase, and CINAHL. We also used Google Scholar to confirm missing studies. The last search was carried out in the beginning of January 2020. Three sets of search terms were used: environment variables (e.g., workplace, worksite, and neighbourhood), physical activity (physical activity and walking), and sedentary behaviour (sedentary behaviour and prolonged sitting). Supplementary Material 1 shows all the search terms and syntax used for the search.

\section{Screening}

The database search produced 2077 articles after removing duplicates. They were screened by two independent reviewers (CYL and YL). The inclusion criteria were: i) published after 2000 in peer-reviewed journals; ii) fulltext was written in English; iii) observational studies with quantitative analyses, and iv) estimated associations of self-reported or objective built environmental measures around the workplace with physical activity or sedentary behaviour among workers. We conducted the review on articles published after the year 2000 because studies on this topic began to emerge around that time [10]. Those studies which examined only other environmental measures, for example, the social (e.g., organisational support), informational (e.g., posters or programs), and interior (e.g., workstations) environments, which were not incorporated with neighbourhood workplace built environment were excluded. We targeted workers who mostly conduct sedentary desk-based work in a primary work location (e.g., office workers), so studies that focused on factory workers, drivers, and clinical nurses were excluded. The screening process based on title and abstract removed 1945 articles. Two independent reviewers (CYL and YL) read the full text of the remaining 132 articles to check their eligibility. This process identified 55 articles to be included in the review by the two reviewers [14-68]. The consistency of the screening process between the two reviewers was over 95\%. Any uncertainty of inclusion of articles was discussed with a third reviewer (MJK) until consensus was reached. 


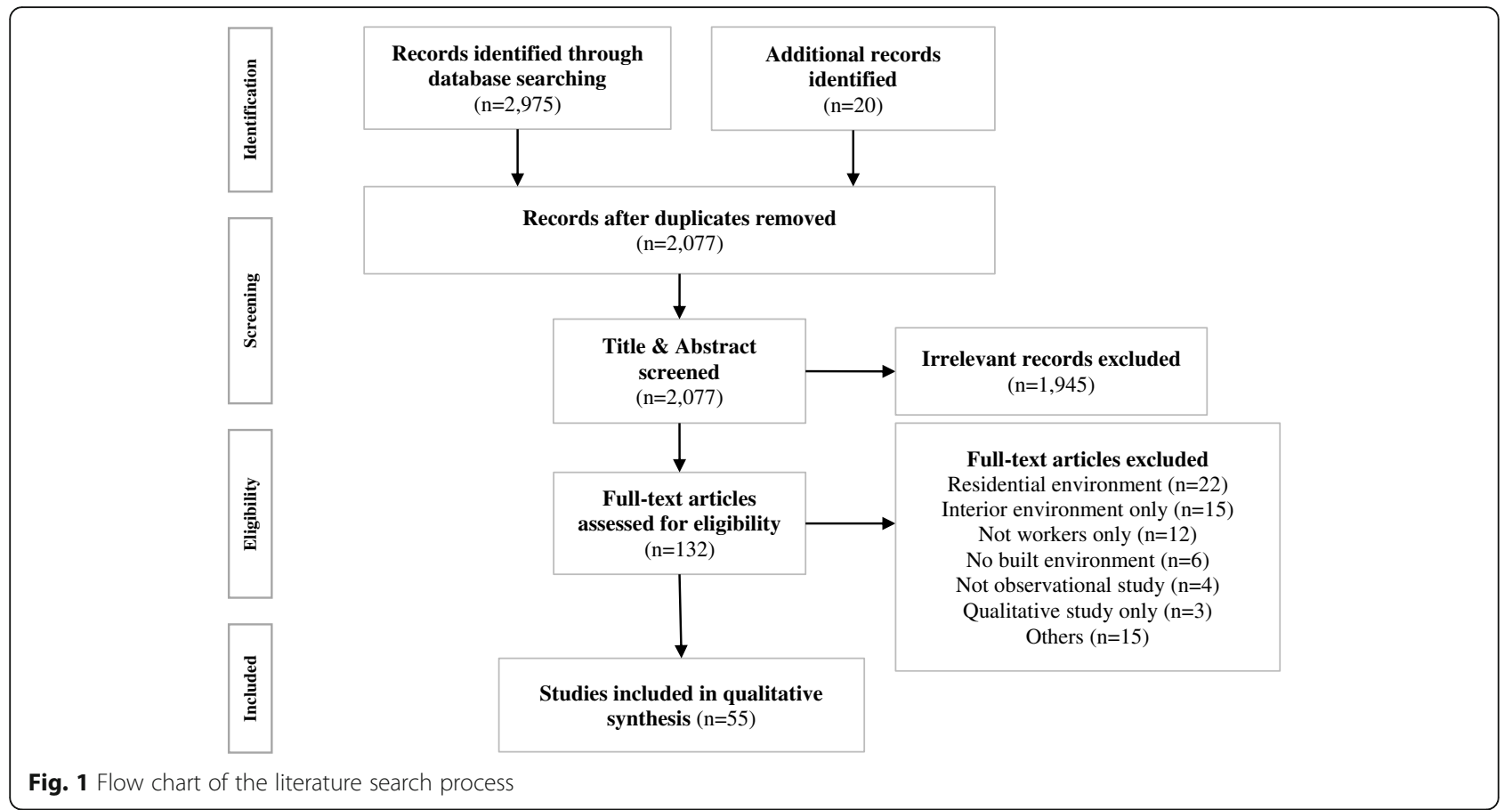

Figure 1 shows the flow chart illustrating the process of database search and screening.

\section{Data extraction}

All relevant information from the full-texts of the eligible articles was extracted by one reviewer (CYL) and crosschecked by the second reviewer (YL). We extracted the following information: study location; sample; study design; built environment attributes and measurement methods (i.e., perceived or objectively-measured); outcome variables and measurement methods (i.e., reported or objectivelymeasured); covariates; and results. Associations between built environment attributes and outcome variables were reported in various ways, including Spearman or Pearson correlation coefficients, regression beta coefficients, and odds ratios.

The outcome variables were categorised into total and different domains of physical activity and sedentary behaviour, including occupational; transport; and recreational physical activity or sedentary behaviour, based on the definitions employed in each study.

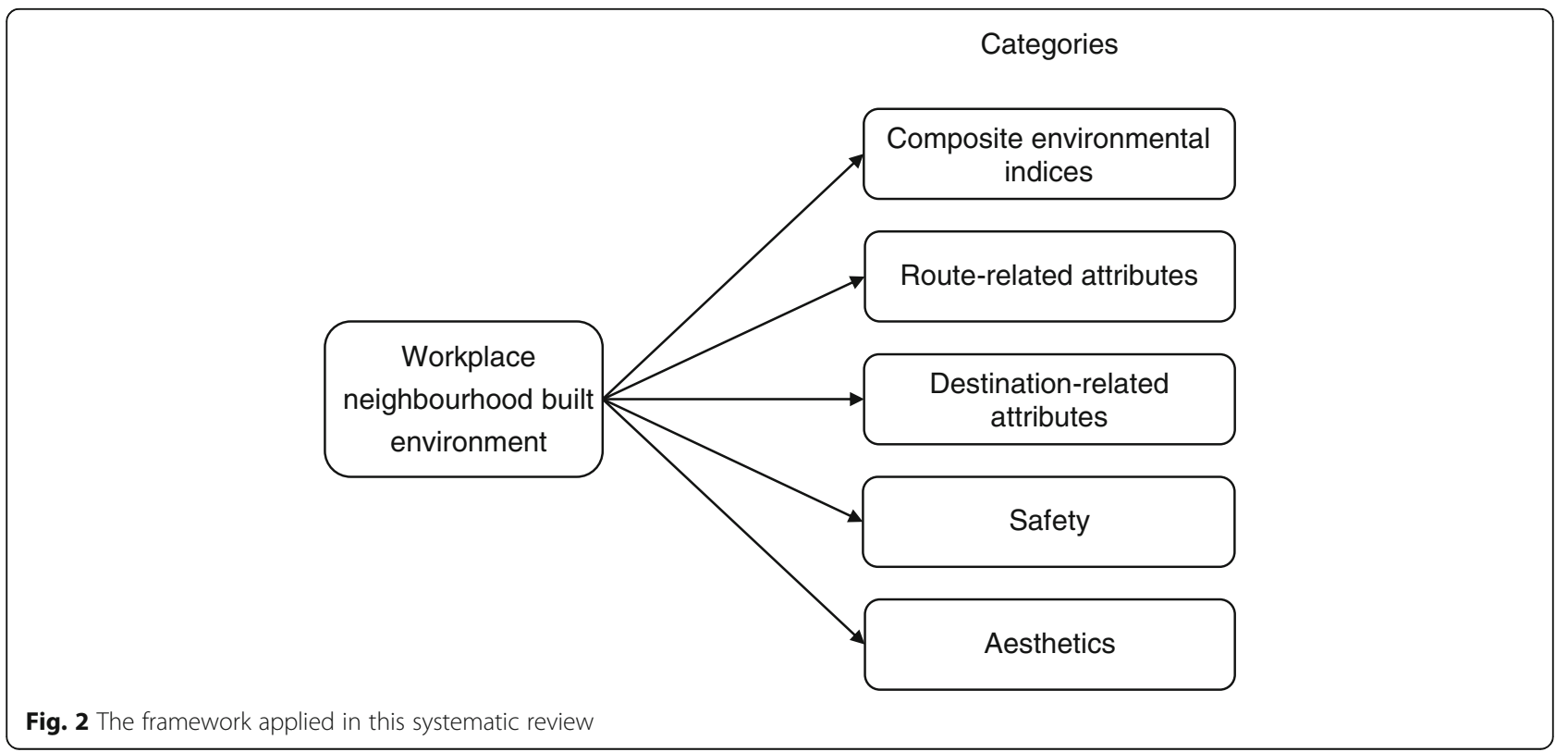


Adapting the categories of neighbourhood built environment attributes employed in the previous reviews [11, 12, 69], we divided the workplace neighbourhood built environment into five categories (Fig. 2):

i) Composite environmental indices: a) a composite index including multiple neighbourhood built environments across different types (e.g., walkability calculating the density, land use mix, and connectivity); and, b) a composite index mixing neighbourhood built environment with other attributes such as interior built environment and/or workplace policies together (e.g., a scale measuring facilities around workplace, social climate, and organisational supports).

ii) Route-related attributes: these included routes for pedestrians or cyclists and street connectivity or intersection density.

iii) Destination-related attributes: these included the presence, density, and diversity of destinations which were assumed to increase physical activity such as shops, transport stops, recreational facilities; and the distance between workplace and home or city centre. Of note, the presence of car parking, which was specifically examined as a matter of convenience to drive cars (i.e., a type of sedentary transport behaviour), was also examined in some studies.

iv) Safety: these comprised a low volume of traffic for pedestrians and bicyclists, low crime rates, and lighting along the commuting routes.

v) Aesthetics: these included general aesthetics, greenness, and being free of litter.

\section{Quality assessment}

The scientific rigour of the selected articles was reviewed and assessed by two independent reviewers (CYL and $\mathrm{YL}$ ), using the study quality assessment tool for observational cohort and cross-sectional studies [70]. The details of this assessment tool are shown in Supplementary Material 2. Each article was assessed against 14 criteria, including research aim, participants, measurements, and statistical analysis. Based on the guidance for the assessment tool [70], the research aim was assessed based on its importance and explicit description. Detailed information such as the demographic, location, and inclusion criteria provided showing a higher probability to replicate the study. Studies using a reliable and valid measurement of variables, employing multiple measurements, and considering the potential covariates typically receive higher quality scores. Each article was given a quality rating of good, fair, or poor according to the scoring guidelines. Disagreements were discussed between the two raters until consensus was reached. Most of the reviewed studies were of good (58.2\%) or fair (40.0\%) quality; therefore, we did not weight study findings based on their rigour. There was moderate agreement between the two independent raters on the quality assessment of the included studies; the percentage of overall agreement was $85.5 \%$, and Cohen's Kappa coefficient was 0.69 .

\section{Synthesis of research findings}

The associations of workplace built environment attributes with physical activity, or sedentary behaviour were coded into "+" (significant positive association), "-" (significant negative association), and "N" (non-significant association). If a study reported findings from several models, only the results of the most adjusted models were used. If a study reported findings from a composite score as well as its components, the results of each component were extracted. Furthermore, if a study showed results for both the overall sample and subsamples, the results from the subsamples were primarily extracted. We summarised the findings for each domain of physical activity and sedentary behaviour separately. This review considered an association to be significant if the $p$-value of an observed effect was $<0.05$.

\section{Results}

\section{Study characteristics}

Cross-sectional studies accounted for $85.5 \%$ of the 55 included articles (Table 1). The period of follow-up implemented in the eight longitudinal studies ranged from 1 month $[20,67,68]$ to 7 years [59]. Around half of the studies reviewed $(n=26)$ were included in the recent review by Zhu et al. [10], with the other half of the studies $(n=29)$ being novel to our review. Most of the studies were undertaken in the USA $(n=25,45.5 \%)$ and the UK ( $n=13,23.6 \%$ ), accounting for around $70 \%$ of all studies.

All of the studies reviewed sampled working adults while some studies examined the associations in question for particular population subgroups such as women [38, 63], parents [24, 50], specific nationalities [19, 36, 51, 67, 68], commuters $[34,46]$, and workers working in an university [28] and local governments [65]. Sample sizes ranged from 26 to 111,808 , of which three had a sample size lower than $100[23,53,67]$ and five with a sample size larger than 10 , $000[36,51,58,61,65]$.

Among the 55 studies reviewed, most investigated physical activity $(n=52)$ rather than sedentary behaviour $(n=7)$; four investigated both physical activity and sedentary behaviour. The transport domain was most commonly examined in relation to both physical activity (40 out of 52 studies) and sedentary behaviour (7 out of 7 studies). Nearly half of the studies used validated outcome measures, including accelerometers $[14,21,23,40$, $53,60,62,63]$ and questionnaires with acceptable 
Table 1 Characteristics and findings of observational studies $(n=55)$ examining associations of workplace neighbourhood built environment attributes with physical activity/sedentary behaviour

\begin{tabular}{|c|c|c|c|c|c|c|}
\hline $\begin{array}{l}\text { The lead } \\
\text { author } \\
\text { (Year) }\end{array}$ & Sample & $\begin{array}{l}\text { Sample and Study } \\
\text { design }\end{array}$ & $\begin{array}{l}\text { Built environment } \\
\text { attributes }\end{array}$ & $\mathrm{PA}$ and $\mathrm{SB}$ & $\begin{array}{l}\text { Results of } \\
\text { the most } \\
\text { adjusted } \\
\text { models }\end{array}$ & Covariates \\
\hline $\begin{array}{l}\text { Adams } \\
\text { (2016) [49] }\end{array}$ & $\begin{array}{l}676 \\
\text { employed } \\
\text { adults, UK }\end{array}$ & $\begin{array}{l}\text { Recruited employees } \\
\text { through five } \\
\text { employers in England; } \\
\text { Cross-sectional }\end{array}$ & $\begin{array}{l}\text { i. Walking routes } \\
\text { (Route-related; P) } \\
\text { ii. Walking pavements } \\
\text { (Route-related; P) } \\
\text { iii. Maintained } \\
\text { pavements (Route- } \\
\text { related; P) } \\
\text { iv. Safe to cross the } \\
\text { road (Safety; P) } \\
\text { v. Dangerous traffic } \\
\text { for walking (Safety; P) } \\
\text { vi. Crime rate } \\
\text { (Safety; P) } \\
\text { vii. Routes are well lit } \\
\text { (Safety; P) } \\
\text { viii. Free of litter/ } \\
\text { graffiti (Aesthetics; P) } \\
\text { ix. Walking routes are } \\
\text { well signposted } \\
\text { (Safety; P) } \\
\text { x. Public transport } \\
\text { (Destination- } \\
\text { related; P) }\end{array}$ & $\begin{array}{l}\text { a. Time spent walking } \\
\text { to and from work } \\
\text { (Transport PA; R) }\end{array}$ & $\begin{array}{l}\text { i-a. + } \\
\text { ii-a. + } \\
\text { iii-a. + } \\
\text { iv-a. N } \\
\text { v-a. N } \\
\text { vi-a. N } \\
\text { vii-a. N } \\
\text { viii-a. N } \\
\text { ix-a. N } \\
\text { x-a. }+\end{array}$ & $\begin{array}{l}\text { Sex, age, car } \\
\text { ownership, distance } \\
\text { lived from work, free } \\
\text { car parking at work, } \\
\text { and organisation }\end{array}$ \\
\hline $\begin{array}{l}\text { Adams } \\
\text { (2017) [55] }\end{array}$ & $\begin{array}{l}1544 \\
\text { employed } \\
\text { adults, UK }\end{array}$ & $\begin{array}{l}\text { Recruited employees } \\
\text { through five } \\
\text { employers in England; } \\
\text { Cross-sectional }\end{array}$ & $\begin{array}{l}\text { i. Distance to home } \\
\text { (Destination-related; } \\
\text { P) }\end{array}$ & $\begin{array}{l}\text { a. Time spent walking } \\
\text { to and from work } \\
\text { (Transport PA; R) }\end{array}$ & i-a. N & $\begin{array}{l}\text { Age, car ownership, } \\
\text { free car parking at } \\
\text { work, work-related PA, } \\
\text { occupation, work pat- } \\
\text { tern, perceived bar- } \\
\text { riers, and psychosocial } \\
\text { factors }\end{array}$ \\
\hline $\begin{array}{l}\text { Adlakha } \\
\text { (2015) [44] }\end{array}$ & $\begin{array}{l}2015 \\
\text { employed } \\
\text { adults, USA }\end{array}$ & $\begin{array}{l}\text { A multistage } \\
\text { sampling frame was } \\
\text { used to randomly } \\
\text { select adults form list- } \\
\text { assisted telephone } \\
\text { random-digit-dialing } \\
\text { methods; } \\
\text { Cross-sectional }\end{array}$ & $\begin{array}{l}\text { i. Healthy restaurants } \\
\text { (Destination-related; } \\
\text { P) } \\
\text { ii. Transit stop } \\
\text { (Destination-related; } \\
\text { P) } \\
\text { iii. Sidewalks (Route- } \\
\text { related; P) } \\
\text { iv. Shops, stores, or } \\
\text { markets (Destination- } \\
\text { related; P) } \\
\text { V. Facilities to bicycle } \\
\text { (Route-related; P) } \\
\text { vi. Recreation facilities } \\
\text { (Destination-related; } \\
\text { P) } \\
\text { vii. Crime rate } \\
\text { (Safety; P) } \\
\text { viii. Dangerous traffic } \\
\text { for pedestrian (Safety; } \\
\text { P) }\end{array}$ & $\begin{array}{l}\text { a. Work PA } \\
\text { (Occupational PA; R) } \\
\text { b. Travel PA } \\
\text { (Transport PA; R) } \\
\text { c. Leisure PA } \\
\text { (Recreational PA; R) } \\
\text { d. Total PA (Total } \\
\text { PA; R) }\end{array}$ & $\begin{array}{l}\text { i-a. N; i-b. +; } \\
\text { i-c. +; i-d. N } \\
\text { ii-a. N; ii-b. N; } \\
\text { ii-c. N; ii-d. + } \\
\text { iii-a. N; iii-b. } \\
\text { N; iii-c. N; } \\
\text { iii-d. + } \\
\text { iv-a. N; iv-b. } \\
\text { +; iv-c. N; iv- } \\
\text { d. N } \\
\text { v-a. +; v-b. +; } \\
\text { v-c. +; v-d. + } \\
\text { vi-a. N; vi-b. } \\
\text { +; vi-c. + } \text {; vi- } \\
\text { d. N } \\
\text { vii-a. - - vii-b. } \\
\text { N; vii-c. N; } \\
\text { vii-d. N } \\
\text { viii-a. N; viii- } \\
\text { b. N; viii-c. N; } \\
\text { viii-d. N }\end{array}$ & $\begin{array}{l}\text { Sex, age, ethnicity, } \\
\text { education, and } \\
\text { income }\end{array}$ \\
\hline $\begin{array}{l}\text { Almeida } \\
\text { (2014) [37] }\end{array}$ & $\begin{array}{l}6261 \\
\text { employed } \\
\text { adults, USA }\end{array}$ & $\begin{array}{l}\text { Recruited employees } \\
\text { in working in } \\
\text { medium-sized work } \\
\text { places in Virginia and } \\
\text { Colorado; Cross- } \\
\text { sectional }\end{array}$ & $\begin{array}{l}\text { i. Outdoor space } \\
\text { (Composite index; O) }\end{array}$ & $\begin{array}{l}\text { a. Moderate activity } \\
\text { and strength-training } \\
\text { activities (Total PA; R) }\end{array}$ & i-a. N & $\begin{array}{l}\text { Sex, age, ethnicity, } \\
\text { and education }\end{array}$ \\
\hline $\begin{array}{l}\text { Badland } \\
\text { (2008) [18] }\end{array}$ & $\begin{array}{l}364 \\
\text { employed } \\
\text { adults not } \\
\text { working }\end{array}$ & $\begin{array}{l}\text { A random sample } \\
\text { selected from electric } \\
\text { telephone white } \\
\text { pages; }\end{array}$ & $\begin{array}{l}\text { i. Residential density } \\
\text { (Destination-related; } \\
\text { O) } \\
\text { ii. Mixed land use }\end{array}$ & $\begin{array}{l}\text { a. Transport-related } \\
\text { PA (Transport PA; R) }\end{array}$ & $\begin{array}{l}\text { i-a. N } \\
\text { ii-a. N } \\
\text { iii-a. + } \\
\text { iv-a. - }\end{array}$ & $\begin{array}{l}\text { Sex, age, ethnicity, } \\
\text { education, household } \\
\text { income, and require } \\
\text { automobile for work }\end{array}$ \\
\hline
\end{tabular}


Table 1 Characteristics and findings of observational studies $(n=55)$ examining associations of workplace neighbourhood built environment attributes with physical activity/sedentary behaviour (Continued)

\begin{tabular}{|c|c|c|c|c|c|c|}
\hline $\begin{array}{l}\text { The lead } \\
\text { author } \\
\text { (Year) }\end{array}$ & Sample & $\begin{array}{l}\text { Sample and Study } \\
\text { design }\end{array}$ & $\begin{array}{l}\text { Built environment } \\
\text { attributes }\end{array}$ & PA and SB & $\begin{array}{l}\text { Results of } \\
\text { the most } \\
\text { adjusted } \\
\text { models }\end{array}$ & Covariates \\
\hline & $\begin{array}{l}\text { from home, } \\
\text { New } \\
\text { Zealand }\end{array}$ & Cross-sectional & $\begin{array}{l}\text { (Destination-related; } \\
\text { O) } \\
\text { iii. Street connectivity } \\
\text { (Route-related; O) } \\
\text { iv. Distance to home } \\
\text { (Destination-related; } \\
\text { O) }\end{array}$ & & & \\
\hline $\begin{array}{l}\text { Badland } \\
(2010) \text { [22] }\end{array}$ & $\begin{array}{l}1188 \\
\text { employed } \\
\text { adults not } \\
\text { working } \\
\text { from home, } \\
\text { New } \\
\text { Zealand }\end{array}$ & $\begin{array}{l}\text { A random sample } \\
\text { selected from electric } \\
\text { telephone white } \\
\text { pages; Cross-sectional }\end{array}$ & $\begin{array}{l}\text { i. Car parking } \\
\text { (Destination-related; } \\
\text { P) } \\
\text { ii. Workplace located } \\
\text { in an urban area } \\
\text { (Destination-related; } \\
\text { O) } \\
\text { iii. Distance to home } \\
\text { (Destination-related; } \\
\text { O) }\end{array}$ & $\begin{array}{l}\text { a. Commuting to } \\
\text { work by public } \\
\text { transport (Transport } \\
\text { PA; R) }\end{array}$ & $\begin{array}{l}\text { i-a. - } \\
\text { ii-a. + } \\
\text { iii-a. N }\end{array}$ & $\begin{array}{l}\text { Sex, age, sample } \\
\text { weighting, residential } \\
\text { accessibility to public } \\
\text { transport, access to } \\
\text { private automobile, } \\
\text { current driving } \\
\text { license, and require } \\
\text { automobile for work }\end{array}$ \\
\hline $\begin{array}{l}\text { Barrington } \\
(2015)[45]\end{array}$ & $\begin{array}{l}1007 \\
\text { employed } \\
\text { adults, USA }\end{array}$ & $\begin{array}{l}\text { Recruited employees } \\
\text { working in the Seattle } \\
\text { area through } \\
\text { workplaces; } \\
\text { Longitudinal (follow- } \\
\text { up: } 2 \text { years) }\end{array}$ & $\begin{array}{l}\text { i. Intersections (Route- } \\
\text { related; O) } \\
\text { ii. Residential units } \\
\text { (Destination-related; } \\
\text { O) } \\
\text { iii. Food destinations } \\
\text { (Destination-related; } \\
\text { O) } \\
\text { iv. Activity } \\
\text { destinations } \\
\text { (Destination-related; } \\
\text { O) }\end{array}$ & $\begin{array}{l}\text { a. Total free-time PA } \\
\text { (Recreational PA; R) } \\
\text { b. Total walking (Total } \\
\text { PA; R) }\end{array}$ & $\begin{array}{l}\text { i-a. N; i-b. N } \\
\text { ii-a. N; ii-b. + } \\
\text { iii-a. NR; iii-b. } \\
\text { N } \\
\text { iv-a. N; iv-b. } \\
\text { N }\end{array}$ & $\begin{array}{l}\text { Sex, age, ethnicity, } \\
\text { education, household } \\
\text { income, manual } \\
\text { occupation, } \\
\text { intervention arm, } \\
\text { worksite parcel size, } \\
\text { and worksite internal } \\
\text { environment } \\
\text { variables, and } \\
\text { worksite SES }\end{array}$ \\
\hline $\begin{array}{l}\text { Batista Ferrer } \\
(2018) \text { [60] }\end{array}$ & $\begin{array}{l}654 \\
\text { employed } \\
\text { adults, UK }\end{array}$ & $\begin{array}{l}\text { A convenience } \\
\text { sample of employees } \\
\text { was recruited from } 87 \\
\text { workplaces in urban } \\
\text { areas in England and } \\
\text { Wales; } \\
\text { Cross-sectional }\end{array}$ & $\begin{array}{l}\text { i. Distance to home } \\
\text { (Destination-related; } \\
\text { O) } \\
\text { ii. Perceptions of the } \\
\text { commuting } \\
\text { environment } \\
\text { (Composite index; P) }\end{array}$ & $\begin{array}{l}\text { a. Incorporating PA } \\
\text { during the commute } \\
\text { (Transport PA; O) } \\
\text { b. Commuting to } \\
\text { work by walking } \\
\text { (Transport PA; O) } \\
\text { c. Commuting to } \\
\text { work by public } \\
\text { transport (Transport } \\
\text { PA; O) }\end{array}$ & $\begin{array}{l}\text { i-a. -; i-b. -; } \\
\text { i-c. NR } \\
\text { ii-a. NR; ii-b. } \\
\text { NR; ii-c. + }\end{array}$ & $\begin{array}{l}\text { BMI and occupational } \\
\text { activity (for a) } \\
\text { Workplace, access to } \\
\text { car, and availability of } \\
\text { workplace car parking } \\
\text { (for b) } \\
\text { Age, access to car, } \\
\text { workplace, availability } \\
\text { of workplace car } \\
\text { parking, and } \\
\text { combines commute } \\
\text { with caring } \\
\text { responsibilities (for c) }\end{array}$ \\
\hline $\begin{array}{l}\text { Biswas } \\
(2018)[61]\end{array}$ & $\begin{array}{l}60,650 \\
\text { employed } \\
\text { adults, } \\
\text { Canada }\end{array}$ & $\begin{array}{l}\text { A multistage } \\
\text { sampling frame was } \\
\text { used to select } \\
\text { households across } \\
\text { Canada randomly; } \\
\text { Cross-sectional }\end{array}$ & $\begin{array}{l}\text { i. Combination of all } \\
\text { (Composite index; P) } \\
\text { ii. Combination of } \\
\text { walking and playing } \\
\text { place (Composite } \\
\text { index; P) } \\
\text { iii. Combination of } \\
\text { walking place, gym, } \\
\text { fitness class, showers/ } \\
\text { change rooms, and } \\
\text { health programs } \\
\text { (Composite index; P) } \\
\text { iv. Combination of } \\
\text { walking place, } \\
\text { showers/change } \\
\text { rooms, and health } \\
\text { programs (Composite } \\
\text { index; P) } \\
\text { v. Combination of } \\
\text { walking place and }\end{array}$ & $\begin{array}{l}\text { a. Leisure-time PA } \\
\text { (Recreational PA; R) }\end{array}$ & $\begin{array}{l}\text { i-a. }+ \\
\text { ii-a. }+ \\
\text { iii-a. }+ \\
\text { iv-a. }+ \\
\text { v-a. N }\end{array}$ & $\begin{array}{l}\text { Sex, age, ethnicity, } \\
\text { marital status, } \\
\text { immigrant, education, } \\
\text { BMI, dietary intake, } \\
\text { smoker status, alcohol } \\
\text { consumption, } \\
\text { perceived health and } \\
\text { mental health, } \\
\text { income, hours worked } \\
\text { per week, working at } \\
\text { home, job stress, } \\
\text { physical demands of } \\
\text { work, and season }\end{array}$ \\
\hline
\end{tabular}


Table 1 Characteristics and findings of observational studies $(n=55)$ examining associations of workplace neighbourhood built environment attributes with physical activity/sedentary behaviour (Continued)

\begin{tabular}{|c|c|c|c|c|c|c|}
\hline $\begin{array}{l}\text { The lead } \\
\text { author } \\
\text { (Year) }\end{array}$ & Sample & $\begin{array}{l}\text { Sample and Study } \\
\text { design }\end{array}$ & $\begin{array}{l}\text { Built environment } \\
\text { attributes }\end{array}$ & $\mathrm{PA}$ and $\mathrm{SB}$ & $\begin{array}{l}\text { Results of } \\
\text { the most } \\
\text { adjusted } \\
\text { models }\end{array}$ & Covariates \\
\hline & & & $\begin{array}{l}\text { showers/change } \\
\text { rooms (Composite } \\
\text { index; } P \text { ) }\end{array}$ & & & \\
\hline $\begin{array}{l}\text { Bjorkelund } \\
\text { (2016) [50] }\end{array}$ & $\begin{array}{l}709 \\
\text { employed } \\
\text { parents not } \\
\text { working } \\
\text { from home, } \\
\text { Norway }\end{array}$ & $\begin{array}{l}\text { Recruited employed } \\
\text { parents of children in } \\
\text { 6th and 7th graders } \\
\text { at } 27 \text { randomly } \\
\text { selected schools in } \\
\text { two Norwegian } \\
\text { counties; } \\
\text { Cross-sectional }\end{array}$ & $\begin{array}{l}\text { i. Distance to home } \\
\text { (Destination-related; } \\
\text { P) } \\
\text { ii. Traffic safety (Safety; } \\
\text { P) }\end{array}$ & $\begin{array}{l}\text { a. Walking to work } \\
\text { (Transport PA; R) } \\
\text { b. Cycling to work } \\
\text { (Transport PA; R) } \\
\text { c. Driving to work } \\
\text { (Transport SB; R) }\end{array}$ & $\begin{array}{l}\text { i-a. }-; \text { i-b. -; } \\
\text { i-c. }+ \\
\text { ii-a. +; ii-b. +; } \\
\text { ii-c. N }\end{array}$ & $\begin{array}{l}\text { Sex, education, } \\
\text { ethnicity, access to } \\
\text { cars/bikes, and } \\
\text { attitudes (for a and b) } \\
\text { Sex, education, } \\
\text { ethnicity, access to } \\
\text { cars/bikes, attitudes, } \\
\text { and leisure-time PA } \\
\text { (for c) }\end{array}$ \\
\hline $\begin{array}{l}\text { Bopp (2012) } \\
\text { [29] }\end{array}$ & $\begin{array}{l}375 \\
\text { employed } \\
\text { adults, USA }\end{array}$ & $\begin{array}{l}\text { Recruited local } \\
\text { employees working in } \\
\text { Manhattan, Kansas, } \\
\text { through community } \\
\text { listservs, links from } \\
\text { local websites, and } \\
\text { fliers; } \\
\text { Cross-sectional }\end{array}$ & $\begin{array}{l}\text { i. Travel time to home } \\
\text { (Destination-related; } \\
\text { P) } \\
\text { ii. Lack of sidewalks } \\
\text { (Route-related; P) } \\
\text { iii. Difficult terrain } \\
\text { (Safety; P) }\end{array}$ & $\begin{array}{l}\text { a. Walking to work } \\
\text { (Transport PA; R) } \\
\text { b. Biking to work } \\
\text { (Transport PA; R) } \\
\text { c. Driving to work } \\
\text { (Transport SB; R) }\end{array}$ & $\begin{array}{l}\text { i-a. -; i-b. N; } \\
\text { i-c. }+ \\
\text { ii-a. N; ii-b. N; } \\
\text { ii-c. N } \\
\text { iii-a. N; iii-b. } \\
\text { N; iii-c. N }\end{array}$ & $\begin{array}{l}\text { Sex, age, ethnicity, } \\
\text { education, self- } \\
\text { efficacy, ecological } \\
\text { friendly attitude, em- } \\
\text { ployment level, occu- } \\
\text { pation classification, } \\
\text { employment length, } \\
\text { perceptions of co- } \\
\text { worker's active, bar- } \\
\text { riers, and motivations }\end{array}$ \\
\hline $\begin{array}{l}\text { Bopp (2013) } \\
\text { [31] }\end{array}$ & $\begin{array}{l}1234 \\
\text { employed } \\
\text { adults not } \\
\text { working } \\
\text { from home, } \\
\text { USA }\end{array}$ & $\begin{array}{l}\text { A convenience } \\
\text { sample was recruited } \\
\text { in medium-large cities } \\
\text { in the mid-Atlantic re- } \\
\text { gion of the U.S. } \\
\text { through email ad- } \\
\text { dresses directly or list- } \\
\text { serv, e-newsletter, or } \\
\text { mass email; } \\
\text { Cross-sectional }\end{array}$ & $\begin{array}{l}\text { i. Lack of bike lanes } \\
\text { (Route-related; P) } \\
\text { ii. Lack of walking/ } \\
\text { biking paths (Route- } \\
\text { related; P) } \\
\text { iii. Lack of sidewalks } \\
\text { (Route-related; P) } \\
\text { iv. Traffic volume } \\
\text { (Safety; P) } \\
\text { v. Crime level (Safety; } \\
\text { P) } \\
\text { vi. Difficult terrain } \\
\text { (Safety; P) } \\
\text { vii. Distance to home } \\
\text { (Destination-related; } \\
\text { P) }\end{array}$ & $\begin{array}{l}\text { a. Work-related active } \\
\text { commuting (Transport } \\
\text { PA; R) }\end{array}$ & $\begin{array}{l}\text { i-a. N } \\
\text { ii-a. N } \\
\text { iii-a. N } \\
\text { iv-a. N } \\
\text { v-a. N } \\
\text { vi-a. N } \\
\text { vii-a. N }\end{array}$ & $\begin{array}{l}\text { Sex, age, ethnicity, } \\
\text { marital status, BMI, } \\
\text { number of children, } \\
\text { number of chronic } \\
\text { disease, active } \\
\text { commuting beliefs, } \\
\text { perceived behavioral } \\
\text { control, self-efficacy, } \\
\text { income, employment } \\
\text { categories, employ- } \\
\text { ment length, number } \\
\text { of cars in the house- } \\
\text { hold, social support, } \\
\text { and residential } \\
\text { environments }\end{array}$ \\
\hline $\begin{array}{l}\text { Bopp (2014) } \\
\text { [38] }\end{array}$ & $\begin{array}{l}709 \\
\text { employed } \\
\text { women not } \\
\text { working } \\
\text { from home, } \\
\text { USA }\end{array}$ & $\begin{array}{l}\text { A convenience } \\
\text { sample was recruited } \\
\text { in medium-large cities } \\
\text { in the mid-Atlantic re- } \\
\text { gion of the U.S. } \\
\text { through email ad- } \\
\text { dresses directly or list- } \\
\text { serv, e-newsletter, or } \\
\text { mass email; } \\
\text { Cross-sectional }\end{array}$ & $\begin{array}{l}\text { i. Lack of bike lanes } \\
\text { (Route-related; P) } \\
\text { ii. Lack of walking/ } \\
\text { biking paths (Route- } \\
\text { related; P) } \\
\text { iii. Lack of sidewalks } \\
\text { (Route-related; P) } \\
\text { iv. Traffic volume } \\
\text { (Safety; P) } \\
\text { v. Crime level (Safety; } \\
\text { P) } \\
\text { vi. Difficult terrain } \\
\text { (Safety; P) } \\
\text { vii. Travel time to } \\
\text { home (Destination- } \\
\text { related; P) }\end{array}$ & $\begin{array}{l}\text { a. Active commuting } \\
\text { to work (Transport PA; } \\
\text { R) }\end{array}$ & $\begin{array}{l}\text { i-a. N } \\
\text { ii-a. N } \\
\text { iii-a. N } \\
\text { iv-a. N } \\
\text { v-a. N } \\
\text { vi-a. N } \\
\text { vii-a. N }\end{array}$ & $\begin{array}{l}\text { Age, number of } \\
\text { chronic diseases, } \\
\text { perceived health } \\
\text { status, self-efficacy, } \\
\text { and perceived behav- } \\
\text { ioral control }\end{array}$ \\
\hline $\begin{array}{l}\text { Bopp (2014) } \\
\text { [39] }\end{array}$ & $\begin{array}{l}997 \\
\text { employed } \\
\text { adults not } \\
\text { working } \\
\text { from home, } \\
\text { USA }\end{array}$ & $\begin{array}{l}\text { A convenience } \\
\text { sample was recruited } \\
\text { in medium-large cities } \\
\text { in the mid-Atlantic re- } \\
\text { gion of the U.S. } \\
\text { through email ad- } \\
\text { dresses directly or }\end{array}$ & $\begin{array}{l}\text { i. Lack of bike lanes } \\
\text { (Route-related; } P \text { ) } \\
\text { ii. Lack of walking/ } \\
\text { biking paths (Route- } \\
\text { related; } P \text { ) } \\
\text { iii. Lack of sidewalks } \\
\text { (Route-related; } P \text { ) }\end{array}$ & $\begin{array}{l}\text { a. Active commuting } \\
\text { to and from work } \\
\text { (Transport PA; R) }\end{array}$ & $\begin{array}{l}\text { Older adults } \\
\text { i-a. N } \\
\text { ii-a. N } \\
\text { iii-a. N } \\
\text { iv-a. N } \\
\text { v-a. NR } \\
\text { vi-a. N }\end{array}$ & $\begin{array}{l}\text { Sex, BMI, number of } \\
\text { children, number of } \\
\text { chronic diseases, } \\
\text { number of cars in the } \\
\text { household, self- } \\
\text { efficacy, perceived be- } \\
\text { havioral control, }\end{array}$ \\
\hline
\end{tabular}


Table 1 Characteristics and findings of observational studies $(n=55)$ examining associations of workplace neighbourhood built environment attributes with physical activity/sedentary behaviour (Continued)

\begin{tabular}{|c|c|c|c|c|c|c|}
\hline $\begin{array}{l}\text { The lead } \\
\text { author } \\
\text { (Year) }\end{array}$ & Sample & $\begin{array}{l}\text { Sample and Study } \\
\text { design }\end{array}$ & $\begin{array}{l}\text { Built environment } \\
\text { attributes }\end{array}$ & PA and SB & $\begin{array}{l}\text { Results of } \\
\text { the most } \\
\text { adjusted } \\
\text { models }\end{array}$ & Covariates \\
\hline & & $\begin{array}{l}\text { listserv, e-newsletter, } \\
\text { or mass email; } \\
\text { Cross-sectional }\end{array}$ & $\begin{array}{l}\text { iv. Traffic volume } \\
\text { (Safety; P) } \\
\text { v. Crime level (Safety; } \\
\text { P) } \\
\text { vi. Difficult terrain } \\
\text { (Safety; P) } \\
\text { vii. Travel time to } \\
\text { home (Destination- } \\
\text { related; P) }\end{array}$ & & $\begin{array}{l}\text { vii-a. - } \\
\text { Younger } \\
\text { adults } \\
\text { i-a. N } \\
\text { ii-a. N } \\
\text { iii-a. N } \\
\text { iv-a. N } \\
\text { v-a. N } \\
\text { vi-a. N } \\
\text { vii-a. N }\end{array}$ & $\begin{array}{l}\text { perceived health sta- } \\
\text { tus, behavioral beliefs, } \\
\text { marital status, ethni- } \\
\text { city, income, educa- } \\
\text { tion, employment, } \\
\text { social support, and } \\
\text { residential } \\
\text { environment }\end{array}$ \\
\hline $\begin{array}{l}\text { Buehler } \\
\text { (2012) [30] }\end{array}$ & $\begin{array}{l}5091 \\
\text { employed } \\
\text { adults, USA }\end{array}$ & $\begin{array}{l}\text { A national survey to } \\
\text { recruit a random } \\
\text { sample of address list- } \\
\text { based households; } \\
\text { Cross-sectional }\end{array}$ & $\begin{array}{l}\text { i. Distance to home } \\
\text { (Destination-related; } \\
\text { P) }\end{array}$ & $\begin{array}{l}\text { a. Biking to work } \\
\text { (Transport PA; R) }\end{array}$ & i-a. - & $\begin{array}{l}\text { Sex, age, ethnicity, } \\
\text { household income, } \\
\text { access to cars/bikes, } \\
\text { residential population } \\
\text { density, residential } \\
\text { area, bikeway supply, } \\
\text { season, and } \\
\text { workplace policies }\end{array}$ \\
\hline $\begin{array}{l}\text { Carlson } \\
\text { (2018) [62] }\end{array}$ & $\begin{array}{l}1085 \\
\text { employed } \\
\text { adults not } \\
\text { working } \\
\text { from home, } \\
\text { USA }\end{array}$ & $\begin{array}{l}\text { Employees were } \\
\text { selected randomly } \\
\text { from households } \\
\text { systematically } \\
\text { selected to vary in } \\
\text { land use patterns and } \\
\text { income; } \\
\text { Cross-sectional }\end{array}$ & $\begin{array}{l}\text { i. Land use mix } \\
\text { (Destination-related; } \\
\text { P) } \\
\text { ii. Street connectivity } \\
\text { (Route-related; P) } \\
\text { iii. Walking/cycling } \\
\text { facilities (Route- } \\
\text { related; P) } \\
\text { iv. Aesthetics; } \\
\text { (Aesthetics; P) } \\
\text { v. Traffic safety (Safety; } \\
\text { P) } \\
\text { vi. Pedestrian safety } \\
\text { (Safety; P) } \\
\text { vii. Crime safety } \\
\text { (Safety; P) }\end{array}$ & $\begin{array}{l}\text { a. Total active } \\
\text { transport (Transport } \\
\text { PA; R) } \\
\text { b. Active transport } \\
\text { around work } \\
\text { (Transport PA; R) } \\
\text { c. Active transport to/ } \\
\text { from work (Transport } \\
\text { PA; R) } \\
\text { d. Total MVPA (Total } \\
\text { PA; O) }\end{array}$ & $\begin{array}{l}\text { i-a. +; i-b. +; } \\
\text { i-c. +; i-d. N } \\
\text { ii-a. N; ii-b. +; } \\
\text { ii-c. N; ii-d. N } \\
\text { iii-a. N; iii-b. } \\
\text { N; iii-c. +; iii- } \\
\text { d. N } \\
\text { iv-a. N; iv-b. } \\
\text { N; iv-c. N; iv- } \\
\text { d. N } \\
\text { v-a. N; v-b. } \\
\text { +; v-c. +; v-d. } \\
\text { N } \\
\text { vi-a. N; vi-b. } \\
\text { +; vi-c. N; vi- } \\
\text { d. + } \\
\text { vii-a. -; vii-b. } \\
\text {-; vii-c. N; } \\
\text { vii-d. + }\end{array}$ & $\begin{array}{l}\text { Sex, age, education, } \\
\text { ethnicity, vehicles per } \\
\text { adult, marital status, } \\
\text { people per } \\
\text { household, time at } \\
\text { address, city, and } \\
\text { clustering of } \\
\text { participants within } \\
\text { block groups }\end{array}$ \\
\hline $\begin{array}{l}\text { Christiansen } \\
\text { (2017) [56] }\end{array}$ & $\begin{array}{l}4764 \\
\text { employed } \\
\text { adults, } \\
\text { Norway }\end{array}$ & $\begin{array}{l}\text { A national survey was } \\
\text { randomly sampled } \\
\text { among residents in } \\
\text { each county in } \\
\text { Norway; } \\
\text { Cross-sectional }\end{array}$ & $\begin{array}{l}\text { i. Distance to home } \\
\text { (Destination-related; } \\
\text { P) } \\
\text { ii. Limited parking } \\
\text { availability } \\
\text { (Destination-related; } \\
\text { P) } \\
\text { iii. Land use mix } \\
\text { (Destination-related; } \\
\text { O) } \\
\text { iv. Distance to the city } \\
\text { centre (Destination- } \\
\text { related; O) }\end{array}$ & $\begin{array}{l}\text { a. Trip from home to } \\
\text { work by car } \\
\text { (Transport SB; R) }\end{array}$ & $\begin{array}{l}\text { i-a. + } \\
\text { ii-a. - } \\
\text { iii-a. - } \\
\text { iv-a. + }\end{array}$ & $\begin{array}{l}\text { Age, education, } \\
\text { household income, } \\
\text { and residential } \\
\text { environment }\end{array}$ \\
\hline $\begin{array}{l}\text { Clark (2016) } \\
\text { [51] }\end{array}$ & $\begin{array}{l}\text { 15,200 } \\
\text { employed } \\
\text { English } \\
\text { adults, UK }\end{array}$ & $\begin{array}{l}\text { A national survey of } \\
\text { multistage sampling } \\
\text { and the same } \\
\text { individuals are re- } \\
\text { interviewed in each } \\
\text { wave; } \\
\text { Longitudinal (follow- } \\
\text { up: } 1 \text { year) }\end{array}$ & $\begin{array}{l}\text { i. Distance to home } \\
\text { (Destination-related; } \\
\text { P) } \\
\text { ii. Change in the } \\
\text { distance to home } \\
\text { between wave } 1 \text { and } \\
\text { wave } 2 \text { (Destination- } \\
\text { related; P) }\end{array}$ & $\begin{array}{l}\text { a. Car commuting to } \\
\text { work (Transport SB; R) } \\
\text { b. Active commuting } \\
\text { to work (Transport PA; } \\
\text { R) } \\
\text { c. Commute mode } \\
\text { switch from car to } \\
\text { non-car (Transport PA; } \\
\text { R) } \\
\text { d. Commute mode } \\
\text { switch from non-car } \\
\text { to car (Transport SB; }\end{array}$ & $\begin{array}{l}\text { i-a. +; i-b. -; } \\
\text { i-c. NR; i-d. } \\
\text { NR; i-e. NR; i- } \\
\text { f. NR } \\
\text { ii-a. NR; ii-b. } \\
\text { NR; ii-c. -; ii- } \\
\text { d. +; ii-e. +; ii- } \\
\text { f. - }\end{array}$ & $\begin{array}{l}\text { Sex, age, education, } \\
\text { employment type, } \\
\text { household income, } \\
\text { attitudes, household } \\
\text { car ownership, current } \\
\text { driving license, and } \\
\text { residential } \\
\text { environment (for a-b) } \\
\text { Sex, age, education, } \\
\text { employment type, } \\
\text { household income, } \\
\text { attitudes, household }\end{array}$ \\
\hline
\end{tabular}


Table 1 Characteristics and findings of observational studies $(n=55)$ examining associations of workplace neighbourhood built environment attributes with physical activity/sedentary behaviour (Continued)

\begin{tabular}{|c|c|c|c|c|c|c|}
\hline $\begin{array}{l}\text { The lead } \\
\text { author } \\
\text { (Year) }\end{array}$ & Sample & $\begin{array}{l}\text { Sample and Study } \\
\text { design }\end{array}$ & $\begin{array}{l}\text { Built environment } \\
\text { attributes }\end{array}$ & $\mathrm{PA}$ and $\mathrm{SB}$ & $\begin{array}{l}\text { Results of } \\
\text { the most } \\
\text { adjusted } \\
\text { models }\end{array}$ & Covariates \\
\hline
\end{tabular}

\begin{tabular}{|c|c|c|c|c|c|c|}
\hline & & & & $\begin{array}{l}\text { R) } \\
\text { e. Commute mode } \\
\text { switch from active to } \\
\text { non-active (Transport } \\
\text { SB; R) } \\
\text { f. Commute mode } \\
\text { switch from non- } \\
\text { active to active (Trans- } \\
\text { port PA; R) }\end{array}$ & & $\begin{array}{l}\text { car ownership, current } \\
\text { driving license, } \\
\text { residential } \\
\text { environment, and } \\
\text { change in life events } \\
\text { (for c-f) }\end{array}$ \\
\hline $\begin{array}{l}\text { Dalton } \\
\text { (2013) [32] }\end{array}$ & $\begin{array}{l}1124 \\
\text { employed } \\
\text { adults, UK }\end{array}$ & $\begin{array}{l}\text { Recruited employees } \\
\text { working in Cambridge } \\
\text { through workplaces; } \\
\text { Cross-sectional }\end{array}$ & $\begin{array}{l}\text { i. Distance to the } \\
\text { nearest bus stop } \\
\text { (Destination-related; } \\
\text { O) } \\
\text { ii. Distance to the } \\
\text { nearest railway station } \\
\text { (Destination-related; } \\
\text { O) } \\
\text { iii. Number of bus } \\
\text { stops (Destination- } \\
\text { related; O) } \\
\text { iv. Number of } \\
\text { destinations in } \\
\text { working area } \\
\text { (Destination-related; } \\
\text { O) } \\
\text { v. Distance to home } \\
\text { (Destination-related; } \\
\text { O) }\end{array}$ & $\begin{array}{l}\text { a. Public transport use } \\
\text { to work (Transport PA; } \\
\text { R) } \\
\text { b. Biking to work } \\
\text { (Transport PA; R) } \\
\text { c. Walking to work } \\
\text { (Transport PA; R) }\end{array}$ & $\begin{array}{l}\text { i-a. N; i-b. N; } \\
\text { i-c. N } \\
\text { ii-a. N; ii-b. N; } \\
\text { ii-c. N } \\
\text { iii-a. N; iii-b. } \\
\text { N; iii-c. N } \\
\text { iv-a. +; iv-b. } \\
\text { +; iv-c. N } \\
\text { v-a. N; v-b. -; } \\
\text { v-c. - }\end{array}$ & $\begin{array}{l}\text { Sex, age, limiting } \\
\text { illness, deprivation, } \\
\text { education, children in } \\
\text { household, car } \\
\text { ownership, type of } \\
\text { work, residential } \\
\text { environment, and car } \\
\text { parking availability }\end{array}$ \\
\hline $\begin{array}{l}\text { de Geus } \\
\text { (2008) [19] }\end{array}$ & $\begin{array}{l}343 \\
\text { employed } \\
\text { Flemish } \\
\text { adults not } \\
\text { working } \\
\text { from home, } \\
\text { Belgium }\end{array}$ & $\begin{array}{l}\text { Recruited employees } \\
\text { via newsletter } \\
\text { distributed in Flanders } \\
\text { and contacted local } \\
\text { cycle communities for } \\
\text { having enough } \\
\text { cyclists; } \\
\text { Cross-sectional }\end{array}$ & $\begin{array}{l}\text { i. Traffic danger } \\
\text { (Safety; P) } \\
\text { ii. Bicycle lanes } \\
\text { (Route-related; P) } \\
\text { iii. Crime rate (Safety; } \\
\text { P) }\end{array}$ & $\begin{array}{l}\text { a. Cycling for } \\
\text { transport (Transport } \\
\text { PA; R) }\end{array}$ & $\begin{array}{l}\text { i-a. N } \\
\text { ii-a. N } \\
\text { iii-a. N }\end{array}$ & Education \\
\hline $\begin{array}{l}\text { Forsyth } \\
\text { (2014) [40] }\end{array}$ & $\begin{array}{l}446 \\
\text { employed } \\
\text { adults not } \\
\text { working } \\
\text { from home, } \\
\text { USA }\end{array}$ & $\begin{array}{l}\text { A randomly selected } \\
\text { sample of a } \\
\text { residential area at first, } \\
\text { and all households } \\
\text { were invited in the } \\
\text { second stage; } \\
\text { Cross-sectional }\end{array}$ & $\begin{array}{l}\text { i. Housing density } \\
\text { (Destination-related; } \\
\text { O) } \\
\text { ii. Access points } \\
\text { (Destination-related; } \\
\text { O) } \\
\text { iii. Percentage of } \\
\text { commercial land use } \\
\text { (Destination-related; } \\
\text { O) }\end{array}$ & $\begin{array}{l}\text { a. Travel PA (Transport } \\
\text { PA; O) } \\
\text { b. Leisure PA } \\
\text { (Recreational PA; O) } \\
\text { c. Total PA (Total PA; } \\
\text { O) }\end{array}$ & $\begin{array}{l}\text { i-a. +; i-b. N; } \\
\text { i-c. + } \\
\text { ii-a. N; ii-b. N; } \\
\text { ii-c. N } \\
\text { iii-a. +; iii-b. } \\
\text { N; iii-c. N }\end{array}$ & $\begin{array}{l}\text { Sex, age, ethnicity, } \\
\text { education, marital } \\
\text { status, housing } \\
\text { tenure, household } \\
\text { income, household } \\
\text { size, PA at work, and } \\
\text { neighbourhood } \\
\text { clustering }\end{array}$ \\
\hline $\begin{array}{l}\text { Gehrke } \\
\text { (2017) [57] }\end{array}$ & $\begin{array}{l}655 \\
\text { employed } \\
\text { adults, USA }\end{array}$ & $\begin{array}{l}\text { A national survey } \\
\text { randomly sample } \\
\text { household within } \\
\text { Oregon and invite to } \\
\text { participate through } \\
\text { mail and telephone; } \\
\text { Cross-sectional }\end{array}$ & $\begin{array}{l}\text { i. Activity density } \\
\text { (Destination-related; } \\
\text { O) } \\
\text { ii. Employment } \\
\text { density (Destination- } \\
\text { related; O) } \\
\text { iii. Population density } \\
\text { (Destination-related; } \\
\text { O) } \\
\text { iv. Retail density } \\
\text { (Destination-related; } \\
\text { O) } \\
\text { v. Urban living } \\
\text { infrastructure density } \\
\text { (Destination-related; } \\
\text { O) }\end{array}$ & $\begin{array}{l}\text { a. Work-based walking } \\
\text { (Transport PA; R) }\end{array}$ & $\begin{array}{l}\text { i-a. N } \\
\text { ii-a. N } \\
\text { iii-a. + } \\
\text { iv-a. N } \\
\text { v-a. N } \\
\text { vi-a. -, N } \\
\text { vii-a. N, N, N } \\
\text { viii-a. N } \\
\text { ix-a. +, N } \\
\text { x-a. N } \\
\text { xi-a. N, N, N, } \\
\mathrm{N} \\
\text { xii-a. N } \\
\text { xiii-a. N } \\
\text { xiv-a. N } \\
\text { xv-a. N }\end{array}$ & $\begin{array}{l}\text { Sex, employment, and } \\
\text { private vehicle } \\
\text { ownership }\end{array}$ \\
\hline
\end{tabular}


Table 1 Characteristics and findings of observational studies $(n=55)$ examining associations of workplace neighbourhood built environment attributes with physical activity/sedentary behaviour (Continued)

\begin{tabular}{|c|c|c|c|c|c|c|}
\hline $\begin{array}{l}\text { The lead } \\
\text { author } \\
\text { (Year) }\end{array}$ & Sample & $\begin{array}{l}\text { Sample and Study } \\
\text { design }\end{array}$ & $\begin{array}{l}\text { Built environment } \\
\text { attributes }\end{array}$ & $\mathrm{PA}$ and $\mathrm{SB}$ & $\begin{array}{l}\text { Results of } \\
\text { the most } \\
\text { adjusted } \\
\text { models }\end{array}$ & Covariates \\
\hline & & & $\begin{array}{l}\text { vi. Employment } \\
\text { entropy ( } 2 \text { types of } \\
\text { calculation) } \\
\text { (Destination-related; } \\
\text { O) } \\
\text { vii. Employment- } \\
\text { population balance (3 } \\
\text { types of calculation) } \\
\text { (Destination-related; } \\
\text { O) } \\
\text { viii. Block area } \\
\text { (Destination-related; } \\
\text { O) } \\
\text { ix. Block density (2 } \\
\text { types of calculation) } \\
\text { (Destination-related; } \\
\text { O) } \\
\text { x. Connected node } \\
\text { ratio (Route-related; } \\
\text { O) } \\
\text { xi. Connectivity index } \\
\text { (4 types of } \\
\text { calculation) (Route- } \\
\text { related; O) } \\
\text { xii. Cul-de-sac density } \\
\text { (Route-related; O) } \\
\text { xiii. Intersection } \\
\text { density (Route-related; } \\
\text { O) } \\
\text { xiv. Intersection-Cul- } \\
\text { de-sac ratio (Route-re- } \\
\text { lated; O) } \\
\text { xv. The proportion of } \\
\text { local roads (Route- } \\
\text { related; O) } \\
\text { xvi. The proportion of } \\
\text { primary roads (Route- } \\
\text { related; O) } \\
\text { xvii. The proportion of } \\
\text { secondary roads } \\
\text { (Route-related; O) } \\
\text { diii. Street network } \\
\text { O) }\end{array}$ & & $\begin{array}{l}\text { xvi-a. N } \\
\text { xvii-a. N } \\
\text { xviii-a. N }\end{array}$ & \\
\hline $\begin{array}{l}\text { Hamre } \\
(2014) \text { [41] }\end{array}$ & $\begin{array}{l}4630 \text { full- } \\
\text { time } \\
\text { employed } \\
\text { adults, USA }\end{array}$ & $\begin{array}{l}\text { A national survey to } \\
\text { recruit a random } \\
\text { sample of address list- } \\
\text { based households; } \\
\text { Cross-sectional }\end{array}$ & $\begin{array}{l}\text { i. Distance to home } \\
\text { (Destination-related; } \\
\text { P) }\end{array}$ & $\begin{array}{l}\text { a. Public transport use } \\
\text { to work (Transport PA; } \\
\text { R) } \\
\text { b. Walking to work } \\
\text { (Transport PA; R) } \\
\text { c. Cycling to work } \\
\text { (Transport PA; R) }\end{array}$ & $\begin{array}{l}\text { i-a. N; i-b. -; } \\
\text { i-c. - }\end{array}$ & $\begin{array}{l}\text { Sex, age, ethnicity, } \\
\text { household income, } \\
\text { access to cars/bikes, } \\
\text { residential population } \\
\text { density, residential } \\
\text { area, transit access, } \\
\text { bikeway supply, } \\
\text { season, and worksite } \\
\text { policies }\end{array}$ \\
\hline $\begin{array}{l}\text { Handy } \\
\text { (2011) [25] }\end{array}$ & $\begin{array}{l}420 \\
\text { employed } \\
\text { adults not } \\
\text { working } \\
\text { from home, } \\
\text { USA }\end{array}$ & $\begin{array}{l}\text { A random sample of } \\
\text { residents for each of } \\
\text { the six communities } \\
\text { in the U.S.; } \\
\text { Cross-sectional }\end{array}$ & $\begin{array}{l}\text { i. Distance to home } \\
\text { (Destination-related; } \\
\text { P) } \\
\text { ii. Dangerous for } \\
\text { bicycling (Safety; P) }\end{array}$ & $\begin{array}{l}\text { a. Commuting to } \\
\text { work by bicycle } \\
\text { (Transport PA; R) }\end{array}$ & $\begin{array}{l}\text { i-a. - } \\
\text { ii-a. N }\end{array}$ & $\begin{array}{l}\text { Sex, housing tenure, } \\
\text { biking comfort, } \\
\text { commuting beliefs } \\
\text { and preference }\end{array}$ \\
\hline $\begin{array}{l}\text { Heinen } \\
\text { (2013) [33] }\end{array}$ & $\begin{array}{l}4171 \\
\text { employed } \\
\text { adults, }\end{array}$ & $\begin{array}{l}\text { Recruited employees } \\
\text { of large organisations } \\
\text { and residents of }\end{array}$ & $\begin{array}{l}\text { i. Distance to home } \\
\text { (Destination-related; } \\
\text { O) }\end{array}$ & $\begin{array}{l}\text { a. Cycling to work } \\
\text { (Transport PA; R) }\end{array}$ & i-a. - & $\begin{array}{l}\text { Sex, age, ethnicity, } \\
\text { access to cars/ } \\
\text { scooters/bikes, }\end{array}$ \\
\hline
\end{tabular}


Table 1 Characteristics and findings of observational studies $(n=55)$ examining associations of workplace neighbourhood built environment attributes with physical activity/sedentary behaviour (Continued)

\begin{tabular}{|c|c|c|c|c|c|c|}
\hline $\begin{array}{l}\text { The lead } \\
\text { author } \\
\text { (Year) }\end{array}$ & Sample & $\begin{array}{l}\text { Sample and Study } \\
\text { design }\end{array}$ & $\begin{array}{l}\text { Built environment } \\
\text { attributes }\end{array}$ & PA and SB & $\begin{array}{l}\text { Results of } \\
\text { the most } \\
\text { adjusted } \\
\text { models }\end{array}$ & Covariates \\
\hline & Netherlands & $\begin{array}{l}\text { working age in Delft } \\
\text { and Zwolle; } \\
\text { Cross-sectional }\end{array}$ & & & & $\begin{array}{l}\text { purpose to use, } \\
\text { sampling area, } \\
\text { attitude, social } \\
\text { support, facilities at } \\
\text { work, and work } \\
\text { policies }\end{array}$ \\
\hline $\begin{array}{l}\text { Karusisi } \\
(2014) \text { [42] }\end{array}$ & $\begin{array}{l}4127 \\
\text { employed } \\
\text { adults, } \\
\text { France }\end{array}$ & $\begin{array}{l}\text { Employees were } \\
\text { recruited from a free } \\
\text { medical check-up of- } \\
\text { fered by National } \\
\text { Health Insurance } \\
\text { System; } \\
\text { Cross-sectional }\end{array}$ & $\begin{array}{l}\text { i. Destinations density } \\
\text { around the workplace } \\
\text { (Destination-related; } \\
\text { O) }\end{array}$ & $\begin{array}{l}\text { a. Walking for } \\
\text { transport (Transport } \\
\text { PA; R) }\end{array}$ & i-a. + & $\begin{array}{l}\text { Sex, age, marital } \\
\text { status, education, } \\
\text { occupation, } \\
\text { homeownership, } \\
\text { perceived financial } \\
\text { strain, household } \\
\text { income, and the level } \\
\text { of human } \\
\text { development of the } \\
\text { country of birth }\end{array}$ \\
\hline Li (2018) [15] & $\begin{array}{l}2843 \\
\text { employed } \\
\text { adults, Japan }\end{array}$ & $\begin{array}{l}\text { A prospective cohort } \\
\text { study of local } \\
\text { government workers } \\
\text { in a central part of } \\
\text { Japan; } \\
\text { Cross-sectional }\end{array}$ & $\begin{array}{l}\text { i. Walkability } \\
\text { (Composite index; O) } \\
\text { ii. Number of parks/ } \\
\text { green spaces } \\
\text { (Destination-related; } \\
\text { O) } \\
\text { iii. Number of sports } \\
\text { facilities (Destination- } \\
\text { related; O) }\end{array}$ & $\begin{array}{l}\text { a. Habitual walking } \\
\text { during leisure-time } \\
\text { (Recreational PA; R) } \\
\text { b. Habitual exercise } \\
\text { during leisure-time } \\
\text { (Recreational PA; R) }\end{array}$ & $\begin{array}{l}\text { Men } \\
\text { i-a. N; i-b. N } \\
\text { ii-a. N; ii-b. N } \\
\text { iii-a. N; iii-b. } \\
\text { N } \\
\text { Women } \\
\text { i-a. N; i-b. N } \\
\text { ii-a. N; ii-b. N } \\
\text { iii-a. N; iii-b. } \\
\text { N }\end{array}$ & $\begin{array}{l}\text { Age, education, } \\
\text { marital status, office } \\
\text { worker, BMI, smoking } \\
\text { status, alcohol } \\
\text { consumption, } \\
\text { sleeping hours, eating } \\
\text { breakfast every day, } \\
\text { depression, history of } \\
\text { hypertension or } \\
\text { diabetes, residential } \\
\text { environment }\end{array}$ \\
\hline $\begin{array}{l}\text { Lucove } \\
\text { (2007) [17] }\end{array}$ & $\begin{array}{l}987 \\
\text { employed } \\
\text { adults, USA }\end{array}$ & $\begin{array}{l}\text { A random sample } \\
\text { selected from } \\
\text { residential household } \\
\text { phone numbers; } \\
\text { Cross-sectional }\end{array}$ & $\begin{array}{l}\text { i. A safe place to walk } \\
\text { outside work (Safety; } \\
\text { P) }\end{array}$ & $\begin{array}{l}\text { a. Any leisure-time PA } \\
\text { (Recreational PA; R) } \\
\text { b. Work-break PA } \\
\text { (Recreational PA; R) } \\
\text { c. Overall PA (Total } \\
\text { PA; R) }\end{array}$ & $\begin{array}{l}\text { i-a. N; i-b. N; } \\
\text { i-c. N }\end{array}$ & $\begin{array}{l}\text { Sex, age, ethnicity, } \\
\text { education, physical } \\
\text { disability, and general } \\
\text { health }\end{array}$ \\
\hline $\begin{array}{l}\text { Macdonald } \\
\text { (2019) [64] }\end{array}$ & $\begin{array}{l}513 \\
\text { employed } \\
\text { adults, UK }\end{array}$ & $\begin{array}{l}\text { A random sample } \\
\text { selected from the } \\
\text { electoral roll within } \\
\text { local authority; } \\
\text { Cross-sectional }\end{array}$ & $\begin{array}{l}\text { i. Access to public PA } \\
\text { facilities (Destination- } \\
\text { related; O) } \\
\text { ii. Access to private } \\
\text { PA facilities } \\
\text { (Destination-related; } \\
\text { O) }\end{array}$ & a. PA (Total PA; R) & $\begin{array}{l}\text { i-a. N } \\
\text { ii-a. N }\end{array}$ & $\begin{array}{l}\text { Sex, age, and income } \\
\text { deprivation }\end{array}$ \\
\hline $\begin{array}{l}\text { Mackenbach } \\
(2016)[52]\end{array}$ & $\begin{array}{l}482 \\
\text { employed } \\
\text { adults, New } \\
\text { Zealand }\end{array}$ & $\begin{array}{l}\text { A national survey of } \\
\text { multistage stratified } \\
\text { sampling; } \\
\text { Cross-sectional }\end{array}$ & $\begin{array}{l}\text { i. Population density } \\
\text { (Destination-related; } \\
\text { O) } \\
\text { ii. Housing density } \\
\text { (Destination-related; } \\
\text { O) } \\
\text { iii. Apartment density } \\
\text { (Destination-related; } \\
\text { O) } \\
\text { iv. Job accessibility } \\
\text { (Destination-related; } \\
\text { O) } \\
\text { v. Land use mix } \\
\text { (Destination-related; } \\
\text { O) } \\
\text { vi. Number of bus } \\
\text { stops (Destination- } \\
\text { related; O) } \\
\text { vii. Number of rail } \\
\text { stations (Destination- } \\
\text { related; O) }\end{array}$ & $\begin{array}{l}\text { a. Active commuting } \\
\text { to work (Transport PA; } \\
\text { R) }\end{array}$ & $\begin{array}{l}\text { i-a. N } \\
\text { ii-a. - } \\
\text { iii-a. N } \\
\text { iv-a. + } \\
\text { v-a. + } \\
\text { vi-a. N } \\
\text { vii-a. + }\end{array}$ & $\begin{array}{l}\text { Sex, age, income, } \\
\text { household type, } \\
\text { season, day of the } \\
\text { week, and trip } \\
\text { distance }\end{array}$ \\
\hline
\end{tabular}


Table 1 Characteristics and findings of observational studies $(n=55)$ examining associations of workplace neighbourhood built environment attributes with physical activity/sedentary behaviour (Continued)

\begin{tabular}{|c|c|c|c|c|c|c|}
\hline $\begin{array}{l}\text { The lead } \\
\text { author } \\
\text { (Year) }\end{array}$ & Sample & $\begin{array}{l}\text { Sample and Study } \\
\text { design }\end{array}$ & $\begin{array}{l}\text { Built environment } \\
\text { attributes }\end{array}$ & PA and SB & $\begin{array}{l}\text { Results of } \\
\text { the most } \\
\text { adjusted } \\
\text { models }\end{array}$ & Covariates \\
\hline $\begin{array}{l}\text { Marquet } \\
\text { (2018) [63] }\end{array}$ & $\begin{array}{l}147 \text { full-time } \\
\text { employed } \\
\text { women not } \\
\text { working } \\
\text { from home, } \\
\text { USA }\end{array}$ & $\begin{array}{l}\text { A convenience } \\
\text { sample of women } \\
\text { living in the U.S; } \\
\text { Cross-sectional }\end{array}$ & $\begin{array}{l}\text { i. Walkability } \\
\text { (Composite index; O) } \\
\text { ii. Walk Score } \\
\text { (Composite index; O) } \\
\text { iii. Vegetation index } \\
\text { (Aesthetics; O) }\end{array}$ & $\begin{array}{l}\text { a. Total MVPA while at } \\
\text { work (Occupational } \\
\text { PA; O) } \\
\text { b. Total MVPA around } \\
\text { the workplace } \\
\text { (Occupational PA; O) }\end{array}$ & $\begin{array}{l}\text { i-a. +; i-b. + } \\
\text { ii-a. N; ii-b. N } \\
\text { iii-a. -; iii-b. - }\end{array}$ & $\begin{array}{l}\text { Age, having children, } \\
\text { income, work-home } \\
\text { distance, amount of } \\
\text { non-work PA }\end{array}$ \\
\hline $\begin{array}{l}\text { Marquet } \\
\text { (2019) [14] }\end{array}$ & $\begin{array}{l}119 \\
\text { employed } \\
\text { adults, USA }\end{array}$ & $\begin{array}{l}\text { A multistage } \\
\text { sampling frame was } \\
\text { used to select adults } \\
\text { form list-assisted tele- } \\
\text { phone random-digit- } \\
\text { dialing methods } \\
\text { randomly; } \\
\text { Cross-sectional }\end{array}$ & $\begin{array}{l}\text { i. Perceived walkability } \\
\text { (Composite index; P) } \\
\text { ii. Walkability } \\
\text { (Composite index; O) } \\
\text { iii. Walk Score } \\
\text { (Composite index; O) } \\
\text { iv. Vegetation index } \\
\text { (Aesthetics; O) }\end{array}$ & $\begin{array}{l}\text { a. Active minutes at } \\
\text { work (Occupational } \\
\text { PA; O) }\end{array}$ & $\begin{array}{l}\text { i-a. + } \\
\text { ii-a. N } \\
\text { iii-a. + } \\
\text { iv-a. N }\end{array}$ & $\begin{array}{l}\text { Sex, BMI, income, } \\
\text { work type, residential } \\
\text { walkability, outside } \\
\text { work PA }\end{array}$ \\
\hline $\begin{array}{l}\text { Merom } \\
(2008)[20]\end{array}$ & $\begin{array}{l}794 \\
\text { employed } \\
\text { adults not } \\
\text { working } \\
\text { from home, } \\
\text { Australia }\end{array}$ & $\begin{array}{l}\text { A random sample } \\
\text { selected from electric } \\
\text { telephone white } \\
\text { pages; Longitudinal } \\
\text { (follow-up: } 1 \text { month) }\end{array}$ & $\begin{array}{l}\text { i. Distance to home } \\
\text { (Destination-related; } \\
\text { P) }\end{array}$ & $\begin{array}{l}\text { a. Single-day active } \\
\text { commuting to work } \\
\text { (Transport PA; R) } \\
\text { b. Usual active } \\
\text { commuting to work } \\
\text { (Transport PA; R) }\end{array}$ & i-a. -; i-b. N & $\begin{array}{l}\text { Age, education, } \\
\text { marital status, BMI, } \\
\text { self-efficacy, active } \\
\text { commuting beliefs, } \\
\text { and total PA }\end{array}$ \\
\hline $\begin{array}{l}\text { Panter } \\
\text { (2011) [26] }\end{array}$ & $\begin{array}{l}1164 \\
\text { employed } \\
\text { adults, UK }\end{array}$ & $\begin{array}{l}\text { Recruited employees } \\
\text { working in Cambridge } \\
\text { through workplaces; } \\
\text { Cross-sectional }\end{array}$ & $\begin{array}{l}\text { i. Public transport } \\
\text { (Destination-related; } \\
\text { P) } \\
\text { ii. Little traffic (Safety; } \\
\text { P) } \\
\text { iii. Routes for walking } \\
\text { (Route-related; P) } \\
\text { iv. Safe to cross the } \\
\text { road (Safety; P) } \\
\text { v. Dangerous for } \\
\text { cyclists (Safety; P) } \\
\text { vi. Routes for cycling } \\
\text { (Route-related; P) } \\
\text { vii. Distance to home } \\
\text { (Destination-related; } \\
\text { P) }\end{array}$ & $\begin{array}{l}\text { a. Walking to work } \\
\text { (Transport PA; R) } \\
\text { b. Cycling to work } \\
\text { (Transport PA; R) }\end{array}$ & $\begin{array}{l}\text { With car } \\
\text { availability in } \\
\text { household } \\
\text { i-a. +; i-b. NR } \\
\text { ii-a. -; ii-b. N } \\
\text { iii-a. N; iii-b. } \\
\text { NR } \\
\text { iv-a. N; iv-b. } \\
\text { N } \\
\text { v-a. NR; v-b. } \\
\text { N } \\
\text { vi-a. NR; vi-b. } \\
+ \\
\text { vii-a. -; vii-b. } \\
\text { NR } \\
\text { Without car } \\
\text { availability in } \\
\text { household } \\
\text { i-a. N; i-b. NR } \\
\text { ii-a. N; ii-b. N } \\
\text { iii-a. N; iii-b. } \\
\text { NR } \\
\text { iv-a. N; iv-b. } \\
\text { N } \\
\text { v-a. NR; v-b. } \\
\text { N } \\
\text { vi-a. NR; vi-b. } \\
\text { N } \\
\text { vii-a. -; vii-b. } \\
\text { NR }\end{array}$ & $\begin{array}{l}\text { Sex, current driving } \\
\text { licence, and attitude } \\
\text { of car use (for a) } \\
\text { Sex, education, } \\
\text { weight status, limiting } \\
\text { illness, number of } \\
\text { children, car } \\
\text { ownership, and } \\
\text { attitude of car use (for } \\
\text { b) }\end{array}$ \\
\hline $\begin{array}{l}\text { Panter } \\
\text { (2011) [27] }\end{array}$ & $\begin{array}{l}1279 \\
\text { employed } \\
\text { older adults, } \\
\text { UK }\end{array}$ & $\begin{array}{l}\text { A prospective cohort } \\
\text { of adults who } \\
\text { registered at } 121 \\
\text { General Practices } \\
\text { within Norwich and } \\
\text { surrounding towns; } \\
\text { Cross-sectional }\end{array}$ & $\begin{array}{l}\text { i. Route-length ratio } \\
\text { (Route-related; O) } \\
\text { ii. Main or secondary } \\
\text { road on the route } \\
\text { (Route-related; O) } \\
\text { iii. Land use mix } \\
\text { (Destination-related; } \\
\text { O) } \\
\text { iv. Density of road } \\
\text { traffic accidents } \\
\text { (Safety; O) } \\
\text { v. Distance to home }\end{array}$ & $\begin{array}{l}\text { a. Active commuting } \\
\text { to work (Transport PA; } \\
\text { R) }\end{array}$ & $\begin{array}{l}\text { Men } \\
\text { i-a. N } \\
\text { ii-a. NR } \\
\text { iii-a. NR } \\
\text { iv-a. N } \\
\text { v-a. - } \\
\text { Women } \\
\text { i-a. NR } \\
\text { ii-a. - } \\
\text { iii-a. NR } \\
\text { iv-a.NR } \\
\text { v-a. - }\end{array}$ & $\begin{array}{l}\text { Age, social class, BMI, } \\
\text { habit for walking or } \\
\text { cycling for transport, } \\
\text { and residential urban- } \\
\text { rural status, and resi- } \\
\text { dential road density }\end{array}$ \\
\hline
\end{tabular}


Table 1 Characteristics and findings of observational studies $(n=55)$ examining associations of workplace neighbourhood built environment attributes with physical activity/sedentary behaviour (Continued)

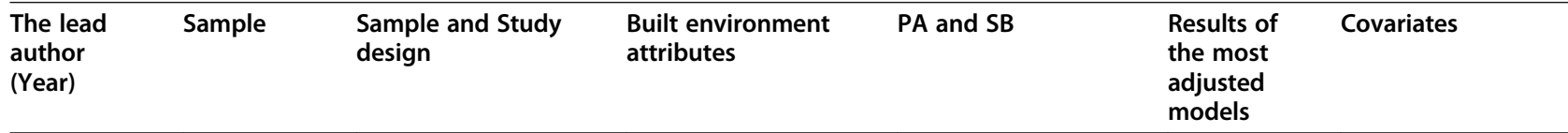

\begin{tabular}{|c|c|c|c|c|c|c|}
\hline & & & $\begin{array}{l}\text { (Destination-related; } \\
\text { O) }\end{array}$ & & & \\
\hline $\begin{array}{l}\text { Panter } \\
\text { (2013) [34] }\end{array}$ & $\begin{array}{l}419 \\
\text { employed } \\
\text { car } \\
\text { commuters } \\
\text { to work, UK }\end{array}$ & $\begin{array}{l}\text { Recruited employees } \\
\text { working in Cambridge } \\
\text { through workplaces; } \\
\text { Cross-sectional }\end{array}$ & $\begin{array}{l}\text { i. Distance to home } \\
\text { (Destination-related; } \\
\text { P) } \\
\text { ii. Supportive } \\
\text { environment } \\
\text { (Composite index; P) }\end{array}$ & $\begin{array}{l}\text { a. Incorporating } \\
\text { walking or cycling } \\
\text { into car journeys to } \\
\text { work (Transport PA; R) }\end{array}$ & $\begin{array}{l}\text { i-a. N } \\
\text { ii-a. }+\end{array}$ & $\begin{array}{l}\text { BMI, work type, } \\
\text { deprivation, } \\
\text { workplace car parking, } \\
\text { attitude towards car, } \\
\text { social norm, and habit } \\
\text { strength for car use }\end{array}$ \\
\hline $\begin{array}{l}\text { Panter } \\
\text { (2013) [35] }\end{array}$ & $\begin{array}{l}655 \\
\text { employed } \\
\text { adults, UK }\end{array}$ & $\begin{array}{l}\text { Recruited employees } \\
\text { working in Cambridge } \\
\text { through workplaces; } \\
\text { Longitudinal (follow- } \\
\text { up: } 1 \text { year) }\end{array}$ & $\begin{array}{l}\text { i. Distance to home } \\
\text { (Destination-related; } \\
\text { P) } \\
\text { ii. Destinations within } \\
\text { walking distance } \\
\text { (Destination-related; } \\
\text { O) } \\
\text { iii. Public transport } \\
\text { (Destination-related; } \\
\text { P) } \\
\text { iv. Little traffic (Safety; } \\
\text { P) } \\
\text { v. Walking routes } \\
\text { (Route-related; P) } \\
\text { vi. Safe to cross the } \\
\text { road (Safety; P) } \\
\text { vii. Dangerous for } \\
\text { cyclists (Safety; P) } \\
\text { viii. Cycling routes } \\
\text { (Route-related; P) }\end{array}$ & $\begin{array}{l}\text { a. Uptake of walking } \\
\text { (Transport PA; R) } \\
\text { b. Uptake of cycling } \\
\text { (Transport PA; R) } \\
\text { c. Uptake of } \\
\text { alternatives to the car } \\
\text { (Transport PA; R) } \\
\text { d. Maintenance of } \\
\text { walking (Transport PA; } \\
\text { R) } \\
\text { e. Maintenance of } \\
\text { cycling (Transport PA; } \\
\text { R) } \\
\text { f. Maintenance of } \\
\text { alternatives to the car } \\
\text { (Transport PA; R) }\end{array}$ & $\begin{array}{l}\text { i-a. N; i-b. N; } \\
\text { i-c. N; i-d. N; } \\
\text { i-e. N; i-f. N } \\
\text { ii-a. N; ii-b. N; } \\
\text { ii-c. N; ii-d. N; } \\
\text { ii-e. N; ii-f. N } \\
\text { iii-a. +; iii-b. } \\
\text { NR; iii-c. N; } \\
\text { iii-d. N; iii-e. } \\
\text { NR; iii-f. N } \\
\text { iv-a. N; iv-b. } \\
\text { N; iv-c. N; iv- } \\
\text { d. N; iv-e. N; } \\
\text { iv-f. N } \\
\text { v-a. N; v-b. } \\
\text { NR; v-c. N; v- } \\
\text { d. N; v-e. NR; } \\
\text { v-f. N } \\
\text { vi-a. N; vi-b. } \\
\text { N; vi-c. N; vi- } \\
\text { d. N; vi-e. N; } \\
\text { vi-f. N } \\
\text { vii-a. NR; vii- } \\
\text { b. N; vii-c. N; } \\
\text { vii-d. NR; vii- } \\
\text { e. N; vii-f. N } \\
\text { viii-a. NR; viii- } \\
\text { b. +; viii-c. +; } \\
\text { viii-d. NR; } \\
\text { viii-e. N; viii- } \\
\text { f. N }\end{array}$ & $\begin{array}{l}\text { Sex, age, weight } \\
\text { status, education, } \\
\text { number of children, } \\
\text { housing tenure, home } \\
\text { location, area-level } \\
\text { deprivation, residential } \\
\text { environment, attitude } \\
\text { to use car, perceived } \\
\text { behaviour control, so- } \\
\text { cial norm, habit } \\
\text { strength, and work- } \\
\text { place car parking }\end{array}$ \\
\hline $\begin{array}{l}\text { Panter } \\
\text { (2014) [43] }\end{array}$ & $\begin{array}{l}655 \\
\text { employed } \\
\text { adults, UK }\end{array}$ & $\begin{array}{l}\text { Recruited employees } \\
\text { working in Cambridge } \\
\text { through workplaces; } \\
\text { Longitudinal (follow- } \\
\text { up: } 1 \text { year) }\end{array}$ & $\begin{array}{l}\text { i. Public transport } \\
\text { (Destination-related; } \\
\text { P) } \\
\text { ii. Little traffic (Safety; } \\
\text { P) } \\
\text { iii. Walking routes } \\
\text { (Route-related; P) } \\
\text { iv. Safe to cross the } \\
\text { road (Safety; P) } \\
\text { v. Dangerous for } \\
\text { cyclists (Safety; P) } \\
\text { vi. Cycling routes } \\
\text { (Route-related; P) }\end{array}$ & $\begin{array}{l}\text { a. Change in time } \\
\text { spent walking on the } \\
\text { commute (Transport } \\
\text { PA; R) } \\
\text { b. Change in time } \\
\text { spent cycling on the } \\
\text { commute (Transport } \\
\text { PA; R) } \\
\text { c. Change in } \\
\text { percentage of car- } \\
\text { only trips on the com- } \\
\text { mute (Transport SB; R) } \\
\text { d. Uptake of walking } \\
\text { on the commute } \\
\text { (Transport PA; R) } \\
\text { e. Uptake of cycling } \\
\text { on the commute } \\
\text { (Transport PA; R) } \\
\text { f. Uptake of an } \\
\text { alternative to the car } \\
\text { on the commute } \\
\text { (Transport PA; R) }\end{array}$ & $\begin{array}{l}\text { i-a. N; i-b. } \\
\text { NR; i-c. N; i- } \\
\text { d. N; i-e. NR; } \\
\text { i-f. + } \\
\text { ii-a. N; ii-b. N; } \\
\text { ii-c. N; ii-d. N; } \\
\text { ii-e. N; ii-f. N } \\
\text { iii-a. N; iii-b. } \\
\text { NR; iii-c. N; } \\
\text { iii-d. N; iii-e. } \\
\text { NR; iii-f. N } \\
\text { iv-a. N; iv-b. } \\
\text { N; iv-c. -; iv- } \\
\text { d. N; iv-e. N; } \\
\text { iv-f. N } \\
\text { v-a. NR; v-b. } \\
\text { N; v-c. +; v-d. } \\
\text { NR; v-e. N; v- } \\
\text { f. - } \\
\text { vi-a. NR; vi-b. } \\
\text { N; vi-c. N; vi- } \\
\text { d. NR; vi-e. } \\
\text { N; vi-f. N }\end{array}$ & $\begin{array}{l}\text { Sex, age, education, } \\
\text { season, housing } \\
\text { tenure, household } \\
\text { composition, access } \\
\text { to cars/bikes, current } \\
\text { driving licence, and } \\
\text { limiting illness }\end{array}$ \\
\hline
\end{tabular}


Table 1 Characteristics and findings of observational studies $(n=55)$ examining associations of workplace neighbourhood built environment attributes with physical activity/sedentary behaviour (Continued)

\begin{tabular}{|c|c|c|c|c|c|c|}
\hline $\begin{array}{l}\text { The lead } \\
\text { author } \\
\text { (Year) }\end{array}$ & Sample & $\begin{array}{l}\text { Sample and Study } \\
\text { design }\end{array}$ & $\begin{array}{l}\text { Built environment } \\
\text { attributes }\end{array}$ & $\mathrm{PA}$ and $\mathrm{SB}$ & $\begin{array}{l}\text { Results of } \\
\text { the most } \\
\text { adjusted } \\
\text { models }\end{array}$ & Covariates \\
\hline $\begin{array}{l}\text { Paul (2019) } \\
{[65]}\end{array}$ & $\begin{array}{l}23,231 \text { full- } \\
\text { time } \\
\text { employed } \\
\text { adults work- } \\
\text { ing in the } \\
\text { U.S. Depart- } \\
\text { ment of the } \\
\text { Interior, USA }\end{array}$ & $\begin{array}{l}\text { Employees working in } \\
\text { the U.S. Department } \\
\text { of the Interior were } \\
\text { emailed an invitation } \\
\text { to participate and } \\
\text { hyperlink to the } \\
\text { survey; } \\
\text { Cross-sectional }\end{array}$ & $\begin{array}{l}\text { i. Distance to home } \\
\text { (Destination-related; } \\
\text { O) } \\
\text { ii. Workplace located } \\
\text { in a non-metro area } \\
\text { (Destination-related; } \\
\text { O) }\end{array}$ & $\begin{array}{l}\text { a. Commuting to } \\
\text { work by walking } \\
\text { (Transport PA; R) } \\
\text { b. Commuting to } \\
\text { work by cycling } \\
\text { (Transport PA; R) } \\
\text { c. Commuting to } \\
\text { work by non-active } \\
\text { mode incorporating } \\
\text { walking/cycling } \\
\text { (Transport PA; R) }\end{array}$ & $\begin{array}{l}\text { i-a. -; i-b. -; } \\
\text { i-c. N } \\
\text { ii-a. +; ii-b. -; } \\
\text { ii-c. - }\end{array}$ & $\begin{array}{l}\text { Sex, age, and } \\
\text { residential } \\
\text { environment }\end{array}$ \\
\hline $\begin{array}{l}\text { Piatkowski } \\
\text { (2015) [46] }\end{array}$ & $\begin{array}{l}2030 \\
\text { employed } \\
\text { bicycling } \\
\text { commuters, } \\
\text { USA }\end{array}$ & $\begin{array}{l}\text { Participants were } \\
\text { drawn from } \\
\text { individuals that sign- } \\
\text { up to receive more in- } \\
\text { formation about "Bike } \\
\text { to Work Day" (BTWD) } \\
\text { online and solicited } \\
\text { via email to } \\
\text { participate; } \\
\text { Cross-sectional }\end{array}$ & $\begin{array}{l}\text { i. Distance to home } \\
\text { (Destination-related; } \\
\text { P) } \\
\text { ii. Street link-to-node } \\
\text { ratio (Route-related; } \\
\text { O) } \\
\text { iii. Intersection density } \\
\text { (Route-related; O) } \\
\text { iv. Safety and } \\
\text { infrastructure } \\
\text { (Composite index; P) } \\
\text { v. Relative } \\
\text { convenience } \\
\text { (Composite index; P) }\end{array}$ & $\begin{array}{l}\text { a. Biking to work on } \\
\text { BTWD (Transport PA; } \\
\text { R) } \\
\text { b. Occasional } \\
\text { commuter (Transport } \\
\text { PA; R) }\end{array}$ & $\begin{array}{l}\text { i-a. -; i-b. -; } \\
\text { ii-a. N; ii-b. N } \\
\text { iii-a. N; iii-b. } \\
\text { N } \\
\text { iv-a. N; iv-b. } \\
\text { N } \\
\text { v-a. -; v-b. - }\end{array}$ & $\begin{array}{l}\text { Sex, age, ethnicity, } \\
\text { household size, } \\
\text { education, household } \\
\text { income, car } \\
\text { availability, attitude } \\
\text { and perception } \\
\text { factors, and residential } \\
\text { environment }\end{array}$ \\
\hline $\begin{array}{l}\text { Pritchard } \\
\text { (2019) [66] }\end{array}$ & $\begin{array}{l}195 \\
\text { employed } \\
\text { adults, } \\
\text { Norway }\end{array}$ & $\begin{array}{l}\text { A fixed sample which } \\
\text { the same group of } \\
\text { participants working } \\
\text { in intra-city work- } \\
\text { places responded to } \\
\text { both surveys; } \\
\text { Cross-sectional }\end{array}$ & $\begin{array}{l}\text { i. Distance to home } \\
\text { (Destination-related; } \\
\text { O) }\end{array}$ & $\begin{array}{l}\text { a. Commuting to } \\
\text { work by public } \\
\text { transport (Transport } \\
\text { SB; R) } \\
\text { b. Commuting to } \\
\text { work by car/ } \\
\text { motorcycle (Transport } \\
\text { SB; R) }\end{array}$ & i-a. +; i-b. + & $\begin{array}{l}\text { Access to car/bicycle, } \\
\text { having children, and } \\
\text { paid parking around } \\
\text { workplace }\end{array}$ \\
\hline $\begin{array}{l}\text { Prodaniuk } \\
\text { (2004) [16] }\end{array}$ & $\begin{array}{l}897 \\
\text { employed } \\
\text { adults, } \\
\text { Canada }\end{array}$ & $\begin{array}{l}\text { Employees working in } \\
\text { three large } \\
\text { organisations located } \\
\text { in Western Canada } \\
\text { were sent a research } \\
\text { invitation within the } \\
\text { internal mail system } \\
\text { of the workplace; } \\
\text { Cross-sectional }\end{array}$ & $\begin{array}{l}\text { i. Perceived Workplace } \\
\text { Environment Scale } \\
\text { (Composite index; P) }\end{array}$ & $\begin{array}{l}\text { a. Workplace PA } \\
\text { (Occupational PA; R) } \\
\text { b. Leisure-time PA } \\
\text { (Recreational PA; R) }\end{array}$ & i-a. +; i-b. + & Workplace \\
\hline $\begin{array}{l}\text { Quinn (2017) } \\
\text { [58] }\end{array}$ & $\begin{array}{l}111,808 \\
\text { employed } \\
\text { adults, USA }\end{array}$ & $\begin{array}{l}\text { A national survey of } \\
\text { random sampling } \\
\text { using a telephone } \\
\text { survey of landline } \\
\text { numbers; } \\
\text { Cross-sectional }\end{array}$ & $\begin{array}{l}\text { i. Distance to home } \\
\text { (Destination-related; } \\
\text { P) } \\
\text { ii. Travel time to } \\
\text { home (Destination- } \\
\text { related; P) }\end{array}$ & $\begin{array}{l}\text { a. Commuting to } \\
\text { work by walking } \\
\text { (Transport PA; R) } \\
\text { b. Commuting to } \\
\text { work by cycling } \\
\text { (Transport PA; R) }\end{array}$ & $\begin{array}{l}\text { i-a. -; i-b. - } \\
\text { ii-a. -; ii-b. - }\end{array}$ & $\begin{array}{l}\text { Sex, age, ethnicity, } \\
\text { education, household } \\
\text { income, and } \\
\text { geographic location }\end{array}$ \\
\hline $\begin{array}{l}\text { Rafferty } \\
\text { (2016) [53] }\end{array}$ & $\begin{array}{l}26 \text { full-time } \\
\text { office } \\
\text { workers, UK }\end{array}$ & $\begin{array}{l}\text { A convenience } \\
\text { sample of employees } \\
\text { at Glasgow } \\
\text { Caledonian University } \\
\text { recruited by email; } \\
\text { Cross-sectional }\end{array}$ & $\begin{array}{l}\text { i. Distance to home } \\
\text { (Destination-related; } \\
\text { O) }\end{array}$ & $\begin{array}{l}\text { a. Number of steps } \\
\text { (Total PA; O) } \\
\text { b. Total time spent in } \\
\text { MVPA (Total PA; O) } \\
\text { c. Steps were taken } \\
\text { during the commute } \\
\text { (Transport PA; O) } \\
\text { d. Time spent in } \\
\text { MVPA during the } \\
\text { commute (Transport } \\
\text { PA; O) }\end{array}$ & $\begin{array}{l}\text { i-a. N; i-b. N; } \\
\text { i-c. N; i-d. N }\end{array}$ & NA \\
\hline $\begin{array}{l}\text { Schoner } \\
\text { (2015) [47] }\end{array}$ & $\begin{array}{l}614 \\
\text { employed }\end{array}$ & $\begin{array}{l}\text { Sample of residents } \\
\text { from five corridors in }\end{array}$ & $\begin{array}{l}\text { i. Distance to home } \\
\text { (Destination-related; }\end{array}$ & $\begin{array}{l}\text { a. Participation in } \\
\text { bicycle commuting to }\end{array}$ & i-a. - & $\begin{array}{l}\text { Age, employment, } \\
\text { residential preference, }\end{array}$ \\
\hline
\end{tabular}


Table 1 Characteristics and findings of observational studies $(n=55)$ examining associations of workplace neighbourhood built environment attributes with physical activity/sedentary behaviour (Continued)

\begin{tabular}{|c|c|c|c|c|c|c|}
\hline $\begin{array}{l}\text { The lead } \\
\text { author } \\
\text { (Year) }\end{array}$ & Sample & $\begin{array}{l}\text { Sample and Study } \\
\text { design }\end{array}$ & $\begin{array}{l}\text { Built environment } \\
\text { attributes }\end{array}$ & PA and SB & $\begin{array}{l}\text { Results of } \\
\text { the most } \\
\text { adjusted } \\
\text { models }\end{array}$ & Covariates \\
\hline & adults, USA & $\begin{array}{l}\text { the U.S.; Cross- } \\
\text { sectional }\end{array}$ & O) & work (Transport PA; R) & & $\begin{array}{l}\text { travel attitudes, and } \\
\text { residential } \\
\text { environment }\end{array}$ \\
\hline $\begin{array}{l}\text { Schwartz } \\
(2009)[21]\end{array}$ & $\begin{array}{l}117 \\
\text { employed } \\
\text { adults, USA }\end{array}$ & $\begin{array}{l}\text { Convenience sample } \\
\text { selected from } 1 \text { zone } \\
\text { in Maryland; } \\
\text { Cross-sectional }\end{array}$ & $\begin{array}{l}\text { i. Without cul-de-sacs } \\
\text { (Route-related; P) } \\
\text { ii. Four-way intersec- } \\
\text { tions (Route-related; } \\
\text { P) } \\
\text { iii. Sidewalks (Route- } \\
\text { related; P) } \\
\text { iv. Bicycle or } \\
\text { pedestrian trails } \\
\text { (Route-related; P) } \\
\text { v. Trees along the } \\
\text { streets (Aesthetics; P) } \\
\text { vi. Free from litter } \\
\text { (Aesthetics; P) } \\
\text { vii. Traffic danger } \\
\text { (Safety; P) } \\
\text { viii. Crosswalks and } \\
\text { pedestrian signals } \\
\text { (Safety; P) }\end{array}$ & $\begin{array}{l}\text { a. Total number of } \\
\text { walking trips taken } \\
\text { from the workplace } \\
\text { (Transport PA; O) } \\
\text { b. Steps were taken at } \\
\text { or near work } \\
\text { (Occupational PA; O) } \\
\text { c. Average weekday } \\
\text { steps (Total PA; O) }\end{array}$ & $\begin{array}{l}\text { i-a. +; i-b. N; } \\
\text { i-c. N } \\
\text { ii-a. N; ii-b. N; } \\
\text { ii-c. N } \\
\text { iii-a. +; iii-b. } \\
\text { N; iii-c. N } \\
\text { iv-a. N; iv-b. } \\
\text { N; iv-c. N } \\
\text { v-a. N; v-b. } \\
\text { N; v-c. N } \\
\text { vi-a. +; vi-b. } \\
\text { N; vi-c. N } \\
\text { vii-a. N; vii-b. } \\
\text { N; vii-c. N } \\
\text { viii-a. +; viii- } \\
\text { b. N; viii-c. N }\end{array}$ & NA \\
\hline $\begin{array}{l}\text { Troped } \\
\text { (2010) [23] }\end{array}$ & $\begin{array}{l}87 \\
\text { employed } \\
\text { adults, USA }\end{array}$ & $\begin{array}{l}\text { A fixed follow-up } \\
\text { sample limited to trail } \\
\text { users; } \\
\text { Cross-sectional }\end{array}$ & $\begin{array}{l}\text { i. Intersection density } \\
\text { (Route-related; O) } \\
\text { ii. Land use mix } \\
\text { (Destination-related; } \\
\text { O) } \\
\text { iii. Residential } \\
\text { population density } \\
\text { (Destination-related; } \\
\text { O) } \\
\text { iv. Housing unit } \\
\text { density (Destination- } \\
\text { related; O) } \\
\text { v. Vegetation index } \\
\text { (Aesthetics; O) }\end{array}$ & $\begin{array}{l}\text { a. MVPA within } 1 \mathrm{~km} \\
\text { of the workplace } \\
\text { (Occupational PA; O) }\end{array}$ & $\begin{array}{l}\text { i-a. N } \\
\text { ii-a. N } \\
\text { iii-a. + } \\
\text { iv-a. + } \\
\text { v-a. N }\end{array}$ & $\begin{array}{l}\text { Sex, age, ethnicity, } \\
\text { and education }\end{array}$ \\
\hline $\begin{array}{l}\text { Umstattd } \\
\text { (2011) [28] }\end{array}$ & $\begin{array}{l}173 \\
\text { university } \\
\text { employees, } \\
\text { USA }\end{array}$ & $\begin{array}{l}\text { A convenience } \\
\text { sample of university } \\
\text { employees; } \\
\text { Cross-sectional }\end{array}$ & $\begin{array}{l}\text { i. Worksite Supportive } \\
\text { Environments for } \\
\text { Active Living Surveys } \\
\text { (Composite index; P) }\end{array}$ & a. MVPA (Total PA; R) & i-a. N & $\begin{array}{l}\text { Sex, age, ethnicity, } \\
\text { health status, position } \\
\text { type, self-regulation, } \\
\text { self-efficacy, and social } \\
\text { support }\end{array}$ \\
\hline $\begin{array}{l}\text { Watts (2013) } \\
{[36]}\end{array}$ & $\begin{array}{l}48,916 \\
\text { employed } \\
\text { Canadian } \\
\text { adults not } \\
\text { working } \\
\text { from home, } \\
\text { Canada }\end{array}$ & $\begin{array}{l}\text { A multistage } \\
\text { sampling frame was } \\
\text { used to select } \\
\text { households across } \\
\text { Canada randomly; } \\
\text { Cross-sectional }\end{array}$ & $\begin{array}{l}\text { i. Access to PA } \\
\text { amenities (Composite } \\
\text { index; P) }\end{array}$ & $\begin{array}{l}\text { a. Leisure-time PA } \\
\text { (Recreational PA; R) }\end{array}$ & i-a. + & $\begin{array}{l}\text { Sex, age, income, and } \\
\text { education }\end{array}$ \\
\hline $\begin{array}{l}\text { Watts (2016) } \\
{[54]}\end{array}$ & $\begin{array}{l}1538 \\
\text { employed } \\
\text { young } \\
\text { adults, USA }\end{array}$ & $\begin{array}{l}\text { Employees were } \\
\text { recruited from the } \\
\text { third wave of a 10- } \\
\text { year longitudinal } \\
\text { study in young } \\
\text { people who pro- } \\
\text { gressed from adoles- } \\
\text { cence to young } \\
\text { adulthood; } \\
\text { Cross-sectional }\end{array}$ & $\begin{array}{l}\text { i. Distance to fitness } \\
\text { facilities (Destination- } \\
\text { related; P) } \\
\text { ii. Distance to home } \\
\text { (Destination-related; } \\
\text { P) }\end{array}$ & $\begin{array}{l}\text { a. MVPA (Total PA; R) } \\
\text { b. Time spent in } \\
\text { walking or biking to } \\
\text { get places (Transport } \\
\text { PA; R) }\end{array}$ & $\begin{array}{l}\text { i-a. N; i-b. NR } \\
\text { ii-a. N; ii-b. - }\end{array}$ & $\begin{array}{l}\text { Age, ethnicity, and } \\
\text { socio-economic status }\end{array}$ \\
\hline $\begin{array}{l}\text { Wen }(2010) \\
{[24]}\end{array}$ & $\begin{array}{l}888 \\
\text { employed } \\
\text { parents not }\end{array}$ & $\begin{array}{l}\text { Employed parents of } \\
\text { students studying in } \\
\text { public primary }\end{array}$ & $\begin{array}{l}\text { i. Public transport } \\
\text { (Destination-related; } \\
\text { P) }\end{array}$ & $\begin{array}{l}\text { a. Travel to work by } \\
\text { car (Transport SB; R) }\end{array}$ & $\begin{array}{l}\text { i-a. - } \\
\text { ii-a. + } \\
\text { iii-a. N }\end{array}$ & $\begin{array}{l}\text { Clustering by the } \\
\text { school and the } \\
\text { within-school }\end{array}$ \\
\hline
\end{tabular}


Table 1 Characteristics and findings of observational studies $(n=55)$ examining associations of workplace neighbourhood built environment attributes with physical activity/sedentary behaviour (Continued)

\begin{tabular}{|c|c|c|c|c|c|c|}
\hline $\begin{array}{l}\text { The lead } \\
\text { author } \\
\text { (Year) }\end{array}$ & Sample & $\begin{array}{l}\text { Sample and Study } \\
\text { design }\end{array}$ & $\begin{array}{l}\text { Built environment } \\
\text { attributes }\end{array}$ & PA and SB & $\begin{array}{l}\text { Results of } \\
\text { the most } \\
\text { adjusted } \\
\text { models }\end{array}$ & Covariates \\
\hline & $\begin{array}{l}\text { working } \\
\text { from home, } \\
\text { Australia }\end{array}$ & $\begin{array}{l}\text { schools located in the } \\
\text { inner west of Sydney } \\
\text { were recruited; Cross- } \\
\text { sectional }\end{array}$ & $\begin{array}{l}\text { ii. Car parking } \\
\text { (Destination-related; } \\
\text { P) } \\
\text { iii. Reputation for a } \\
\text { safe place (Safety; P) } \\
\text { iv. Distance to home } \\
\text { (Destination-related; } \\
\text { P) }\end{array}$ & & iv-a. N & $\begin{array}{l}\text { intraclass correlation } \\
\text { for travel to work by } \\
\text { car }\end{array}$ \\
\hline $\begin{array}{l}\text { Yang (2015) } \\
{[48]}\end{array}$ & $\begin{array}{l}1332 \\
\text { employed } \\
\text { adults not } \\
\text { working } \\
\text { from home, } \\
\text { USA }\end{array}$ & $\begin{array}{l}\text { Multistage stratified } \\
\text { sampling using list- } \\
\text { assisted telephone } \\
\text { random-digit-dialling; } \\
\text { Cross-sectional }\end{array}$ & $\begin{array}{l}\text { i. Healthy restaurants } \\
\text { (Destination-related; } \\
\text { P) } \\
\text { ii. Transit stop } \\
\text { (Destination-related; } \\
\text { P) } \\
\text { iii. Sidewalks (Route- } \\
\text { related; P) } \\
\text { iv. Shops, stores, or } \\
\text { markets (Destination- } \\
\text { related; P) } \\
\text { v. Facilities to bicycle } \\
\text { (Route-related; P) } \\
\text { vi. Recreation facilities } \\
\text { (Destination-related; } \\
\text { P) } \\
\text { vii. Crime rate (Safety; } \\
\text { P) } \\
\text { viii. Dangerous traffic } \\
\text { for pedestrian (Safety; } \\
\text { P) } \\
\text { ix. Distance to home } \\
\text { (Destination-related; } \\
\text { O) }\end{array}$ & $\begin{array}{l}\text { a. Public transport use } \\
\text { (Transport PA; R) } \\
\text { b. Active commuting } \\
\text { (Transport PA; R) }\end{array}$ & $\begin{array}{l}\text { i-a. N; i-b. N } \\
\text { ii-a. N; ii-b. N } \\
\text { iii-a. N; iii-b. } \\
N \\
\text { iv-a. N; iv-b. } \\
N \\
\text { v-a. N; v-b. N } \\
\text { vi-a. N; vi-b. } \\
+ \\
\text { vii-a. N; vii-b. } \\
N \\
\text { viii-a. N; viii- } \\
\text { b. N } \\
\text { ix-a. N; ix-b. } \\
-\end{array}$ & $\begin{array}{l}\text { Sex, age, BMI, } \\
\text { household car } \\
\text { ownership, and } \\
\text { education (for a) } \\
\text { Sex, age, BMl, and } \\
\text { household car } \\
\text { ownership (for b) }\end{array}$ \\
\hline $\begin{array}{l}\text { Yang (2017) } \\
{[59]}\end{array}$ & $\begin{array}{l}2757 \\
\text { employed } \\
\text { adults, UK }\end{array}$ & $\begin{array}{l}\text { Recruited employees } \\
\text { registered at } 121 \\
\text { General Practices } \\
\text { within Norwich and } \\
\text { surrounding towns; } \\
\text { Longitudinal (follow- } \\
\text { up: } 7 \text { years) }\end{array}$ & $\begin{array}{l}\text { i. Distance to home } \\
\text { (Destination-related; } \\
\text { O) } \\
\text { ii. Route length ratio } \\
\text { (Route-related; O) } \\
\text { iii. Main road on the } \\
\text { route (Route-related; } \\
\text { O) } \\
\text { iv. Secondary road on } \\
\text { route (Route-related; } \\
\text { O) } \\
\text { v. Main or secondary } \\
\text { road along route } \\
\text { (Route-related; O) } \\
\text { vi. Number of } \\
\text { streetlights along } \\
\text { route (Safety; O) } \\
\text { vii. Land use mix } \\
\text { (Destination-related; } \\
\text { O) } \\
\text { viii. Density of road } \\
\text { traffic accidents } \\
\text { (Safety; O) } \\
\text { ix. Density of fatal and } \\
\text { serious road traffic } \\
\text { accidents (Safety; O) }\end{array}$ & $\begin{array}{l}\text { a. Uptake of active } \\
\text { commuting (Transport } \\
\text { PA; R) } \\
\text { b. Maintenance of } \\
\text { active commuting } \\
\text { (Transport PA; R) }\end{array}$ & $\begin{array}{l}\text { i-a. -; i-b. - } \\
\text { ii-a. N; ii-b. N } \\
\text { iii-a. N; iii-b. } \\
N \\
\text { iv-a. N; iv-b. } \\
N \\
\text { v-a. -; v-b. - } \\
\text { vi-a. +; vi-b. } \\
\text { NR } \\
\text { vii-a. N; vii-b. } \\
N \\
\text { viii-a. N; viii- } \\
\text { b. N } \\
\text { ix-a. N; ix-b. } \\
\text { N }\end{array}$ & $\begin{array}{l}\text { Sex, age, BMI, and } \\
\text { residential } \\
\text { environment }\end{array}$ \\
\hline $\begin{array}{l}\text { Zhang } \\
\text { (2019) [67] }\end{array}$ & $\begin{array}{l}98 \\
\text { employed } \\
\text { Chinese } \\
\text { adults, China }\end{array}$ & $\begin{array}{l}\text { A convenience } \\
\text { sample recruited from } \\
\text { two-night schools of- } \\
\text { fered by two }\end{array}$ & $\begin{array}{l}\text { i. Residential density } \\
\text { (Destination-related; } \\
\text { P) } \\
\text { ii. Land-use diversity }\end{array}$ & $\begin{array}{l}\text { a. Transport-related } \\
\text { cycling at time } 1 \\
\text { (Transport PA; R) } \\
\text { b. Transport-related }\end{array}$ & $\begin{array}{l}\text { i-a. N; i-b. N } \\
\text { ii-a. N; ii-b. N } \\
\text { iii-a. N; iii-b. } \\
\text { N }\end{array}$ & NA \\
\hline
\end{tabular}


Table 1 Characteristics and findings of observational studies $(n=55)$ examining associations of workplace neighbourhood built environment attributes with physical activity/sedentary behaviour (Continued)

\begin{tabular}{|c|c|c|c|c|c|c|}
\hline $\begin{array}{l}\text { The lead } \\
\text { author } \\
\text { (Year) }\end{array}$ & Sample & $\begin{array}{l}\text { Sample and Study } \\
\text { design }\end{array}$ & $\begin{array}{l}\text { Built environment } \\
\text { attributes }\end{array}$ & $\mathrm{PA}$ and $\mathrm{SB}$ & $\begin{array}{l}\text { Results of } \\
\text { the most } \\
\text { adjusted } \\
\text { models }\end{array}$ & Covariates \\
\hline & & $\begin{array}{l}\text { universities in Beijing } \\
\text { and Shanghai, China; } \\
\text { Longitudinal (follow- } \\
\text { up: } 1 \text { month) }\end{array}$ & $\begin{array}{l}\text { (Destination-related; } \\
\text { P) } \\
\text { iii. Land-use accessibil- } \\
\text { ity (Destination-re- } \\
\text { lated; P) } \\
\text { iv. Street connectivity } \\
\text { (Route-related; P) } \\
\text { V. Aesthetics } \\
\text { (Aesthetics; P) } \\
\text { vi. Walking } \\
\text { infrastructure (Route- } \\
\text { related; P) } \\
\text { vii. Traffic safety } \\
\text { (Safety; P) } \\
\text { viii. Crime safety } \\
\text { (Safety; P) }\end{array}$ & $\begin{array}{l}\text { cycling at time } 2 \\
\text { (Transport PA; R) }\end{array}$ & $\begin{array}{l}\text { iv-a. N; iv-b. } \\
\mathrm{N} \\
\text { v-a. N; v-b. N } \\
\text { vi-a. N; vi-b. } \\
\mathrm{N} \\
\text { vii-a. N; vii-b. } \\
\mathrm{N} \\
\text { viii-a. N; viii- } \\
\text { b. N }\end{array}$ & \\
\hline $\begin{array}{l}\text { Zhang } \\
\text { (2019) [68] }\end{array}$ & $\begin{array}{l}157 \\
\text { employed } \\
\text { Chinese } \\
\text { adults, China }\end{array}$ & $\begin{array}{l}\text { A convenience } \\
\text { sample recruited from } \\
\text { two-night schools of- } \\
\text { fered by two univer- } \\
\text { sities in Beijing and } \\
\text { Shanghai, China; } \\
\text { Longitudinal (follow- } \\
\text { up: } 1 \text { month) }\end{array}$ & $\begin{array}{l}\text { i. Walkability } \\
\text { (Composite index; P) }\end{array}$ & $\begin{array}{l}\text { a. Transport-related } \\
\text { walking at time } 1 \\
\text { (Transport PA; R) } \\
\text { b. Transport-related } \\
\text { walking at time } 2 \\
\text { (Transport PA; R) }\end{array}$ & i-a. N; i-b. N & $\begin{array}{l}\text { Sex, age, marital } \\
\text { status, education, } \\
\text { number of children, } \\
\text { BMI, income level, } \\
\text { and general health }\end{array}$ \\
\hline
\end{tabular}

Note: PA Physical activity, SB Sedentary behaviour, O Objectively-measured, $P$ Perceived, $R$ Reported, + Positive association, $N$ Non-significant association, Negative association, NR Not reported, NA Not applicable, BMI Body mass index, MVPA Moderate-to-vigorous physical activity

reliability and validity $[16,17,21,26-28,31,32,34,36$, $37,44,45,49,50,54,61,64,67,68]$. Notably, 28 studies assessed self-reported physically-active and sedentary behaviours without reporting the reliability or validity of the questions or questionnaires used.

More than half of the studies measured perceived built environments ( $n=29,52.7 \%), 34.5 \%$ of them measured environments objectively, and $12.7 \%$ included both types of measure in their studies. A vague boundary (e.g., near or surrounding the workplace) was the most commonly used as a perceived neighbourhood definition. When buffers were applied to define workplace neighbourhoods, a $400-[40,57,63]$ or $800-m$ radius $[14,40,45,64]$ and the network buffer $[18,23,40,46,64]$ were the most frequently used buffer size and type, respectively.

\section{Built environment correlates of physical activity and sedentary behaviour}

Detailed syntheses of the findings are shown in Table 2. We reported the findings according to "instances" rather than "studies" as most of the studies reported associations of different built environment attributes with multiple domains of physical activity and sedentary behaviours. Overall, there were 455 instances in our analysis, nearly half of instances involved destination-related attributes (193 out of 455), followed by safety (111 out of 455) and route-related attributes (105 out of 455).
Additionally, most of them examined physical activity (431 out of 455), particularly within the transport domain (325 out of 431). Only 24 out of 455 examined sedentary behaviour, and all of them focused on transport settings.

\section{Composite environmental indices}

There were 14 instances where an association was estimated between a workplace neighbourhood composite index and a physical activity outcome. All measured walkability (primarily based on density, land use mix, and street connectivity), with the only exception measuring the presence of facilities and routes for walking through an audit tool [37]. Most of the instances regarding walkability were measured objectively by geographic information systems based on established indices $(n=7)$ and Walk Score ${ }^{\oplus}(n=3)$ while the remainder measured perceived walkability $(n=3)$. Of 14 instances where composite indexes were examined, only four showed positive associations with physical activity [14, 63]; all of which were in relation to the occupational domain. More than $70 \%$ of the instances reported were nonsignificant.

Fifteen instances used composite indices mixing other attributes (e.g., organised sports teams and classes within the workplace) in addition to workplace neighbourhood built environment attributes. The majority of the 
Table 2 Workplace neighbourhood built environment attributes and workers' active and sedentary behaviours: summary of instances

\begin{tabular}{|c|c|c|c|c|c|c|c|c|c|c|c|c|c|c|}
\hline \multirow{2}{*}{$\begin{array}{l}\text { Workplace neighbourhood } \\
\text { built environment attributes }\end{array}$} & \multirow{2}{*}{$\begin{array}{l}\text { Physical } \\
\text { activity and } \\
\text { sedentary } \\
\text { behavior }\end{array}$} & \multicolumn{3}{|c|}{ Good } & \multicolumn{3}{|c|}{ Fair } & \multicolumn{3}{|c|}{ Poor } & \multicolumn{4}{|c|}{ Total } \\
\hline & & + & $\mathrm{N}$ & - & + & $\mathrm{N}$ & - & + & $\mathrm{N}$ & - & + & $\mathrm{N}$ & - & Sum \\
\hline \multirow[t]{12}{*}{ Composite environmental indices } & \multicolumn{14}{|l|}{ Physical activity } \\
\hline & Total & 0 & 1 & 0 & 0 & 1 & 0 & 0 & 0 & 0 & 0 & 2 & 0 & 2 \\
\hline & Occupational & 2 & 2 & 0 & 3 & 1 & 0 & 0 & 0 & 0 & 5 & 3 & 0 & 8 \\
\hline & Transport & 0 & 4 & 2 & 2 & 0 & 0 & 0 & 0 & 0 & 2 & 4 & 2 & 8 \\
\hline & Recreational & 5 & 1 & 0 & 1 & 4 & 0 & 0 & 0 & 0 & 6 & 5 & 0 & 11 \\
\hline & (sub-total) & 7 & 8 & 2 & 6 & 6 & 0 & 0 & 0 & 0 & 13 & 14 & 2 & 29 \\
\hline & \multicolumn{14}{|c|}{ Sedentary behaviour } \\
\hline & Total & 0 & 0 & 0 & 0 & 0 & 0 & 0 & 0 & 0 & 0 & 0 & 0 & 0 \\
\hline & Occupational & 0 & 0 & 0 & 0 & 0 & 0 & 0 & 0 & 0 & 0 & 0 & 0 & 0 \\
\hline & Transport & 0 & 0 & 0 & 0 & 0 & 0 & 0 & 0 & 0 & 0 & 0 & 0 & 0 \\
\hline & Recreational & 0 & 0 & 0 & 0 & 0 & 0 & 0 & 0 & 0 & 0 & 0 & 0 & 0 \\
\hline & (sub-total) & 0 & 0 & 0 & 0 & 0 & 0 & 0 & 0 & 0 & 0 & 0 & 0 & 0 \\
\hline \multirow[t]{12}{*}{ Route-related attributes } & \multicolumn{14}{|l|}{ Physical activity } \\
\hline & Total & 0 & 7 & 0 & 2 & 0 & 0 & 0 & 0 & 0 & 2 & 7 & 0 & 9 \\
\hline & Occupational & 0 & 5 & 0 & 1 & 1 & 0 & 0 & 0 & 0 & 1 & 6 & 0 & 7 \\
\hline & Transport & 10 & 51 & 2 & 2 & 17 & 1 & 0 & 0 & 0 & 12 & 68 & 3 & 83 \\
\hline & Recreational & 0 & 1 & 0 & 1 & 1 & 0 & 0 & 0 & 0 & 1 & 2 & 0 & 3 \\
\hline & (sub-total) & 10 & 64 & 2 & 6 & 19 & 1 & 0 & 0 & 0 & 16 & 83 & 3 & 102 \\
\hline & \multicolumn{14}{|c|}{ Sedentary behaviour } \\
\hline & Total & 0 & 0 & 0 & 0 & 0 & 0 & 0 & 0 & 0 & 0 & 0 & 0 & 0 \\
\hline & Occupational & 0 & 0 & 0 & 0 & 0 & 0 & 0 & 0 & 0 & 0 & 0 & 0 & 0 \\
\hline & Transport & 0 & 2 & 0 & 0 & 1 & 0 & 0 & 0 & 0 & 0 & 3 & 0 & 3 \\
\hline & Recreational & 0 & 0 & 0 & 0 & 0 & 0 & 0 & 0 & 0 & 0 & 0 & 0 & 0 \\
\hline & (sub-total) & 0 & 2 & 0 & 0 & 1 & 0 & 0 & 0 & 0 & 0 & 3 & 0 & 3 \\
\hline \multirow[t]{12}{*}{ Destination-related attributes } & \multicolumn{14}{|l|}{ Physical activity } \\
\hline & Total & 2 & 9 & 0 & 1 & 3 & 0 & 0 & 2 & 0 & 3 & 14 & 0 & 17 \\
\hline & Occupational & 2 & 1 & 0 & 0 & 4 & 0 & 0 & 0 & 0 & 2 & 5 & 0 & 7 \\
\hline & Transport & 37 & 55 & 2 & 22 & 17 & 2 & 0 & 2 & 0 & 59 & 74 & 4 & 137 \\
\hline & Recreational & 0 & 5 & 0 & 2 & 10 & 0 & 0 & 0 & 0 & 2 & 15 & 0 & 17 \\
\hline & (sub-total) & 41 & 70 & 2 & 25 & 34 & 2 & 0 & 4 & 0 & 66 & 108 & 4 & 178 \\
\hline & \multicolumn{14}{|c|}{ Sedentary behaviour } \\
\hline & Total & 0 & 0 & 0 & 0 & 0 & 0 & 0 & 0 & 0 & 0 & 0 & 0 & 0 \\
\hline & Occupational & 0 & 0 & 0 & 0 & 0 & 0 & 0 & 0 & 0 & 0 & 0 & 0 & 0 \\
\hline & Transport & 0 & 1 & 4 & 2 & 1 & 7 & 0 & 0 & 0 & 2 & 2 & 11 & 15 \\
\hline & Recreational & 0 & 0 & 0 & 0 & 0 & 0 & 0 & 0 & 0 & 0 & 0 & 0 & 0 \\
\hline & (sub-total) & 0 & 1 & 4 & 2 & 1 & 7 & 0 & 0 & 0 & 2 & 2 & 11 & 15 \\
\hline \multirow[t]{5}{*}{ Safety } & \multicolumn{14}{|l|}{ Physical activity } \\
\hline & Total & 2 & 4 & 0 & 0 & 2 & 0 & 0 & 0 & 0 & 2 & 6 & 0 & 8 \\
\hline & Occupational & 0 & 2 & 0 & 1 & 1 & 0 & 0 & 0 & 0 & 1 & 3 & 0 & 4 \\
\hline & Transport & 7 & 70 & 4 & 0 & 8 & 0 & 0 & 0 & 0 & 7 & 78 & 4 & 89 \\
\hline & Recreational & 0 & 2 & 0 & 0 & 2 & 0 & 0 & 0 & 0 & 0 & 4 & 0 & 4 \\
\hline
\end{tabular}


Table 2 Workplace neighbourhood built environment attributes and workers' active and sedentary behaviours: summary of instances (Continued)

\begin{tabular}{|c|c|c|c|c|c|c|c|c|c|c|c|c|c|c|}
\hline \multirow{2}{*}{$\begin{array}{l}\text { Workplace neighbourhood } \\
\text { built environment attributes }\end{array}$} & \multirow{2}{*}{$\begin{array}{l}\text { Physical } \\
\text { activity and } \\
\text { sedentary } \\
\text { behavior }\end{array}$} & \multicolumn{3}{|c|}{ Good } & \multicolumn{3}{|c|}{ Fair } & \multicolumn{3}{|c|}{ Poor } & \multicolumn{4}{|c|}{ Total } \\
\hline & & + & $\mathrm{N}$ & - & + & $\mathrm{N}$ & - & + & $\mathrm{N}$ & - & + & $\mathrm{N}$ & - & Sum \\
\hline & (sub-total) & 9 & 78 & 4 & 1 & 13 & 0 & 0 & 0 & 0 & 10 & 91 & 4 & 105 \\
\hline & Sedentary bet & & & & & & & & & & & & & \\
\hline & Total & 0 & 0 & 0 & 0 & 0 & 0 & 0 & 0 & 0 & 0 & 0 & 0 & 0 \\
\hline & Occupational & 0 & 0 & 0 & 0 & 0 & 0 & 0 & 0 & 0 & 0 & 0 & 0 & 0 \\
\hline & Transport & 0 & 2 & 2 & 0 & 2 & 0 & 0 & 0 & 0 & 0 & 4 & 2 & 6 \\
\hline & Recreational & 0 & 0 & 0 & 0 & 0 & 0 & 0 & 0 & 0 & 0 & 0 & 0 & 0 \\
\hline & (sub-total) & 0 & 2 & 2 & 0 & 2 & 0 & 0 & 0 & 0 & 0 & 4 & 2 & 6 \\
\hline \multirow[t]{12}{*}{ Aesthetics } & Physical activi & & & & & & & & & & & & & \\
\hline & Total & 0 & 3 & 0 & 0 & 0 & 0 & 0 & 0 & 0 & 0 & 3 & 0 & 3 \\
\hline & Occupational & 0 & 3 & 2 & 0 & 1 & 0 & 0 & 0 & 0 & 0 & 4 & 2 & 6 \\
\hline & Transport & 1 & 7 & 0 & 0 & 0 & 0 & 0 & 0 & 0 & 1 & 7 & 0 & 8 \\
\hline & Recreational & 0 & 0 & 0 & 0 & 0 & 0 & 0 & 0 & 0 & 0 & 0 & 0 & 0 \\
\hline & (sub-total) & 1 & 13 & 2 & 0 & 1 & 0 & 0 & 0 & 0 & 1 & 14 & 2 & 17 \\
\hline & Sedentary beh & & & & & & & & & & & & & \\
\hline & Total & 0 & 0 & 0 & 0 & 0 & 0 & 0 & 0 & 0 & 0 & 0 & 0 & 0 \\
\hline & Occupational & 0 & 0 & 0 & 0 & 0 & 0 & 0 & 0 & 0 & 0 & 0 & 0 & 0 \\
\hline & Transport & 0 & 0 & 0 & 0 & 0 & 0 & 0 & 0 & 0 & 0 & 0 & 0 & 0 \\
\hline & Recreational & 0 & 0 & 0 & 0 & 0 & 0 & 0 & 0 & 0 & 0 & 0 & 0 & 0 \\
\hline & (sub-total) & 0 & 0 & 0 & 0 & 0 & 0 & 0 & 0 & 0 & 0 & 0 & 0 & 0 \\
\hline
\end{tabular}

Note: + Positive association, $N$ Non-significant association, - Negative association

instances $(n=9)$ found positive associations with physical activity, mostly about the recreational domain. The remainder of instances found either nonsignificant $[28,46,61]$ or negative associations [46] in relation to physical activity.

There were no instances of associations between composite indices and sedentary behaviour.

\section{Route-related attributes}

Most of the instances examined routes for pedestrians or cyclists and street connectivity, as route-related attributes to investigate their associations with physical activity, especially in the transport settings, which accounted for $81 \%$ of the instances. Around $16 \%$ of the 102 instances reported positive associations with physical activity $[18,21,26,35,44,49,62]$. The majority of instances $(n=83)$ were non-significant. However, three instances were negatively associated with physical activity; all of which were in relation to the transport domain $[27,59]$.

All three instances of examining an association between route-related attributes and sedentary behaviour found that routes for pedestrians or cyclists were not associated with sedentary transport behaviour [29, 43].

\section{Destination-related attributes}

The majority of the instances used the presence, density, and diversity of destinations as destination-related attributes in the workplace neighbourhood to examine their associations with physical activity, especially during transport-related contexts, which accounted for $77 \%$ of the instances. Almost $40 \%$ of the 178 instances which examined destination-related attributes were found to be positively associated with physical activity, mostly in relation to the transport domain. Over $60 \%$ of the instances were non-significant. Additionally, four instances were found to be negatively associated with transport physical activity [22, 52, 57, 65]; of which one negative association examined car parking with transport physical activity [22]. The features of these destination-related attributes identified in the positive and negative associations were different. The presence or density of shops, transport stops, and recreational facilities were more identified in the positive associations; by contrast, all of the negative associations identified that longer distance between workplace and home and car parking around workplace were associated with lower levels of transport physical activity.

The majority of instances (11 out of 15) reported negative associations between destinations-related 
attributes and sedentary behaviour; all of which were regarding the transport domain $[24,29,50,51,56,66]$. Most of these destination-related attributes examined were the distance between workplace and home. Furthermore, the only two instances examining car parking showed positive associations with sedentary transport behaviour [24, 56]. The remainder of instances showed non-significant associations [24, 43]. The diversity of destination-related attributes examined in sedentary behaviour was less than in physical activity; a higher proportion of instances examined the distance between workplace and home with sedentary behaviour.

\section{Safety}

The instances in relation to workplace neighbourhood safety mainly measured the traffic (e.g., low volume of traffic for pedestrians and bicyclists) and crime safety (e.g., low crime rates). Less than $10 \%$ of the 105 instances of estimates between safety and physical activity showed positive associations [21, 44, 50, 59, 62]. Over $85 \%$ of the instances were not significant. Also, there were four instances where safety was negatively associated with transport-related physical activity [26, 43, 62].

Most of the instances (4 out of 6) regarding associations of safety with sedentary behaviour showed nonsignificant associations. The remainder of the instances found that perceiving it to be safer to cross the road and cycle was associated with a lower likelihood of car-only trips [43]. All of the instances were examined in the transport settings.

\section{Aesthetics}

All but 3 of the 17 instances examining associations between workplace neighbourhood aesthetics and physical activity were not significant. There was one instance of a positive association between perceptions that streets were free from litter and transport physical activity [21] and two instances of negative associations between objectively measured greenness and occupational physical activity [63].

None of the instances reported indicators of aesthetics with any domains of sedentary behaviour.

\section{Discussion}

This review examined studies of the associations of workplace neighbourhood built environment attributes with active and sedentary behaviours among adults working in occupations involving prolonged indoor sitting. We found most of the instances examining associations between the workplace neighbourhood built environment and physical activity to be non-significant, irrespective of the study quality. Our findings are consistent with a previous review on workplace neighbourhood built environments [10]. This previous review also showed mostly non-significant associations of workplace neighbourhood built environment attributes with physical activity [10]. However, another systematic review on the residential neighbourhood reported consistently positive associations between accessibility to destinations and transport-related walking [12]. These findings suggest that compared with the residential environment, the workplace environment seems to be a less important context for supporting physically-active behaviours. A potential explanation for the weaker associations of workplace neighbourhood built environment attributes with physical activity in comparison with residential neighbourhoods may be due to workers having less autonomy over behavioural decisions during working hours, especially for those who perceive their managers disapprove of absenteeism from desks for walking [71]. Additionally, a lack of time for exercising during typical working hours may be another explanation for the weaker associations [15].

We found that destination-related attributes, notably longer distances between workplace and home, as well as better access to car parking around the workplace, were positively associated with transport-related sedentary behaviour; the sedentary transport behaviour in all the reviewed studies was travelling by car. A previous review on residential neighbourhood built environments found that better access to recreational facilities and public open spaces were negatively associated with transport-related sedentary behaviour [11]. Although varied destination-related attributes were measured in these two reviews, these findings suggest that better access to destinations, except for car parking, may be disincentives for transport-related sedentary behaviour, in both workplace and residential neighbourhoods. A short travelling distance to the workplace may make it more likely that workers undertake active commuting [72] which can replace time spent in car travel. For this reason, it may not matter whether there are wellmaintained pathways or safe routes in or around the workplace neighbourhood if workers live too far from their workplaces to commute actively. Urban design policies on co-locating residential and workplaces together and decreasing car parking spaces around the workplace or moving car parking further away from workplaces may be effective strategies to reduce workers' time spent in car travel.

The difference in the number of included studies between the previous review [10] and our review was mainly explained by the novel studies published after 2018 and additional studies examining specific neighbourhood built environment attributes. Our findings may suggest an authentic lack of an association between workplace built environments and physical activity, or the null findings may be attributable to the 
misperceptions of neighbourhood environments. A previous study reported that those who were less physically active for transport purposes perceived their high walkable residential neighbourhood as low walkable [73]. Such a misperception of neighbourhood environments may attenuate the associations of perceived workplace neighbourhood built environment attributes with active behaviours because most of the reviewed studies measured perceived environmental attributes.

Based on the review findings, there are some research priorities suggested for improving the quality of future relevant studies, as follows.

\section{Conducting research on workplace neighbourhood built environment and sedentary behaviour}

Most of the previous studies examined the relationships of the workplace neighbourhood built environment with workers' physical activity, rather than their sedentary behaviour. Considering the increased proportion of workers in desk-based occupations [74] and the high proportion of sitting time occurring in the workplace $[1$, 2], more attention is needed in investigating sedentary behaviour and workplace neighbourhood built environment correlates. A recent review showed that previous studies on workplace environments and sedentary behaviour mainly focused on the workplace interior environment, such as workstations [10]; however, our findings suggest that some destination-related attributes surrounding workplaces were associated with sitting time among workers. For developing effective approaches to improving workers' health through reducing sitting time, studies on workplace neighbourhood built environments and sedentary behaviour, particularly the domains most likely to be affected (e.g., occupational and transport-related sedentary behaviour), are needed.

\section{Improving measurement and diversity of workplace neighbourhood built environment}

Most previous studies assessed the workplace neighbourhood built environment using perceived measures; however, perceptions of the attributes of neighbourhoods could vary markedly between individuals, regardless of the objective environmental attributes that exist in the workplace neighbourhood. Furthermore, all the reviewed studies identified the shortest commuting route between workplace and home [27, 32, 33] irrespective of workers' transport modes. The shortest commuting route may not represent the routes taken by the individuals. Future research included both perceived, and objective measures of the workplace neighbourhood built environment and monitoring the actual commuting routes (e.g., by global positioning system) for workers is encouraged to clarify their associations with active and sedentary behaviours. Additionally, some of the reviewed studies measured the variety of public open space in the workplace neighbourhood without considering the quality of such attributes. Previous research has shown that the quality of built environment attributes, including destinations such as parks, may be an essential factor to influence individuals' active behaviours $[75,76]$ and thus should be considered. Future research on diverse varieties of public open space around the workplace and multiple measures for accessibility (e.g., the number of public transport stops as well as its frequency) may provide insights that will be relevant to developing effective strategies to promoting workers' physical activity and reducing sedentary behaviour.

\section{Developing a framework for defining the influential buffers of workplace neighbourhood built environments for active and sedentary behaviours}

Many of the reviewed studies did not clearly define the locations or areas of environmental attributes. Some studies used ambiguous terms (e.g., at or around the workplace) without specifying the neighbourhood's boundary [22, 36, 77]. The core rationale for distinguishing interior and neighbourhood contexts of workplaces is its implications for where responsibility for improvements lies - i.e., land/property owners or governments. Furthermore, re-examining the influential buffers of the workplace is essential as it may be smaller than the frequently used buffers (e.g., 400- and 800-m) in research around residences [69] due to limited free time [15] and less autonomy over their behavioural decisions [71] for workers during working hours. Some other studies combined interior facilities and workplace policies [36, 37, 46] while assessing environmental attributes. These additional variables may contribute to stronger associations. Future studies are recommended to develop a framework in specifying the size of the workplace neighbourhood with clear definitions when examining their associations with physical activity or sedentary behaviours.

\section{Enhancing the correspondence between where built environments and behaviours are assessed}

Most of the previous studies did not precisely designate 'where' the active or sedentary behaviours occurred, whereas they assessed built environment attributes surrounding the workplace only. The disparity between these variables may lead to a misinterpretation of workplace neighbourhood built environments due to the contribution of active or sedentary behaviours in non-work contexts to total physical activity. For example, workers who engaged in more light-intensity physical activity during working hours do compensate by doing less active during non-working hours [78]. Therefore, distinguishing the venue and different intensities for these 
behaviours in different contexts could inform detailed information to examine whether the compensation of physical activity or sedentary behaviour occurred. For ascertaining the associations of the workplace neighbourhood on physical activity or sedentary behaviour that occurs during work hours, or during commuting, studies which could identify specific venues (e.g., global positioning system) and timing-specific behaviours (e.g., accelerometer) are suggested.

\section{Considering potential confounding factors}

When considering the workplace environmental correlates of physical activity or sedentary behaviour, some potential covariates should be examined in future research. For example, some geographic attributes attached to locations play an important role when individuals choose where to reside and work [79]. The self-selection of the residence and workplace may moderate the association of environmental attributes with active and sedentary behaviours. Referring to the ecological model, there may be an accumulative effect across different levels of factors. Individual motivations and attitudes, lifestyle preferences, social supports, interior workplace facilities, and workplace health promotion programs, may all contribute to the associations of workplace built environment attributes with workers' physical activity and sedentary behaviour to some extent [45]. However, few of the reviewed studies accounted for factors such as the preference of the workplace while examining the associations in question. Studies considering these additional factors will provide additional evidence for the independent associations of environmental correlates with active or sedentary behaviours.

\section{Implementing research in diverse settings with prospective designs}

Previous studies on workplace environmental correlates of active or sedentary behaviours have been mainly conducted in Western countries such as the USA and the UK. More relevant studies from non-Western countries should be encouraged because different countries or areas have varied behaviour patterns and neighbourhood built environments. For example, there are marked differences in the prevalence of active commuting across regions [80]. The low prevalence of physically-active commuting in Western countries leads to most of these studies investigating how to promote active commuting to or from work (i.e., transport physical activity) but less into other domains. However, studies giving weight to other domains of physical activity and sedentary behaviour may have more contributions to increase the total amount of physical activity and reduce the total time of sedentary behaviour, especially in countries or areas with a relatively high prevalence of active commuting to work. Additionally, future research should use prospective or experimental designs to evaluate whether changes in workplace neighbourhood built environments affect active and sedentary behaviours, rather than crosssectional designs, which form the majority of the existing evidence base.

\section{Conclusions}

Desk-based workers can spend around $80 \%$ of their working hours sedentary and can have limited opportunities for physical activity in and around the workplace. Synthesizing the current research evidence, we found that workers who lived further from their work and who could easily access car parking surrounding the workplace had a higher likelihood of transport-related sedentary behaviour. However, we found that workplace neighbourhood built environments such as route-related attributes, safety, and aesthetics did not appear to be influential for workers' physically-active and sedentary behaviours. Designing mixed-use neighbourhoods where there are opportunities to live close to workplaces as well as have access to a high density of shops, services, and recreational facilities may be useful for reducing workers' sedentary time. Future investigations with improvements in research design and measurements are needed to more deeply understand the impacts of workplace neighbourhood environments on workers' physically-active and sedentary behaviours.

\section{Supplementary information}

The online version contains supplementary material available at https://doi. org/10.1186/s12966-020-01055-x.

Additional file 1: Supplementary Material 1. Search terms and syntax for the literature search.

Additional file 2: Supplementary Material 2. Quality Assessment Tool for Observational Cohort and Cross-Sectional Studies.

\section{Acknowledgements}

Not applicable.

\section{Authors' contributions}

CYL, MJK, and KO conceived the idea of the systematic review. All the authors developed the protocol of this systematic review. CYL, MJK, YL, and $\mathrm{KO}$ drafted the manuscript, led the data extraction, and synthesised the results. All authors made substantial revisions to earlier drafts and approved the final manuscript.

\section{Funding}

TN is supported by a Japan Society for the Promotion of Sciences (KAKENHI Grant 20H00040). GRM is supported by a Canadian Institutes of Health Research Foundations Scheme Grant (FDN-154331). NO is supported by a National Health and Medical Research Council of Australia (NHMRC) Centre of Research Excellence Grant (\#1057608), NHMRC Senior Principal Research Fellowship (\#1118225), and the Victorian Government's Operational Infrastructure Support Program. KO is supported by a Grant-in-Aid for Scientific Research (No. 20H04113) from the Japan Society for the Promotion of Science. The funding bodies have no influence over the design of the review, collection, synthesis, and interpretation of data of the review. 


\section{Availability of data and materials}

Not applicable.

\section{Ethics approval and consent to participate Not applicable.}

\section{Consent for publication}

Not applicable.

\section{Competing interests}

The authors declare no conflicts of interest.

\section{Author details}

'Graduate School of Sport Sciences, Waseda University, 2-579-15 Mikajima, Tokorozawa, Saitama 359-1192, Japan. ${ }^{2}$ Faculty of Sport Sciences, Waseda University, Tokorozawa, Japan. ${ }^{3}$ Melbourne School of Population and Global Health, The University of Melbourne, Melbourne, Australia. ${ }^{4}$ Behavioural Epidemiology Laboratory, Baker Heart \& Diabetes Institute, Melbourne, Australia. ${ }^{5}$ Department of Health Promotion and Health Education, National Taiwan Normal University, Taipei, Taiwan. ${ }^{6}$ Faculty of Health and Sport Sciences, University of Tsukuba, Tsukuba, Japan. ${ }^{7}$ Graduate School of Environmental Studies, Tohoku University, Sendai, Japan. ${ }^{8}$ Department of Community Health Sciences, Cumming School of Medicine, University of Calgary, Calgary, Canada. ${ }^{9}$ Centre for Urban Transitions, Swinburne University of Technology, Melbourne, Australia.

\section{Received: 8 August 2020 Accepted: 9 November 2020}

\section{Published online: 20 November 2020}

\section{References}

1. Parry S, Straker L. The contribution of office work to sedentary behaviour associated risk. BMC Public Health. 2013;13:296.

2. Waters CN, Ling EP, Chu AH, Ng SH, Chia A, Lim YW, et al. Assessing and understanding sedentary behaviour in office-based working adults: a mixedmethod approach. BMC Public Health. 2016;16:360.

3. Sallis JF, Owen N, Fisher E. Ecological models of health behavior. San Francisco: John Wiley \& Sons, Inc; 2015. p. 43-64.

4. Chu AH, Ng SH, Tan CS, Win AM, Koh D, Muller-Riemenschneider F. A systematic review and meta-analysis of workplace intervention strategies to reduce sedentary time in white-collar workers. Obes Rev. 2016;17(5):467-81.

5. Hutcheson AK, Piazza AJ, Knowlden AP. Work site-based environmental interventions to reduce sedentary behavior: a systematic review. Am J Health Promot. 2018;32(1):32-47.

6. Commissaris DA, Huysmans MA, Mathiassen SE, Srinivasan D, Koppes LLJ, Hendriksen IJ. Interventions to reduce sedentary behavior and increase physical activity during productive work: a systematic review. Scand J Work Environ Health. 2016;42(3):181-91.

7. Colenberg $\mathrm{S}$, Jylhä $\mathrm{T}$, Arkesteijn $\mathrm{M}$. The relationship between interior office space and employee health and well-being-a literature review. Build Res Informat. 2020:1:1-15.

8. Saint-Maurice PF, Troiano RP, Berrigan D, Kraus WE, Matthews CE. Volume of light versus moderate-to-vigorous physical activity: Similar benefits for allcause mortality? J Am Heart Assoc. 2018;7(7):e008815.

9. Learnihan V, Van Niel KP, Giles-Corti B, Knuiman M. Effect of scale on the links between walking and urban design. Geogr Res. 2011;49(2):183-91.

10. Zhu X, Yoshikawa A, Qiu L, Lu Z, Lee C, Ory M. Healthy workplaces, active employees: a systematic literature review on impacts of workplace environments on employees' physical activity and sedentary behavior. Build Environ. 2020:168:106455

11. Koohsari MJ, Sugiyama T, Sahlqvist S, Mavoa S, Hadgraft N, Owen N. Neighborhood environmental attributes and adults' sedentary behaviors: review and research agenda. Prev Med. 2015;77:141-9.

12. Sugiyama T, Neuhaus M, Cole R, Giles-Corti B, Owen N. Destination and route attributes associated with adults' walking: a review. Med Sci Sports Exerc. 2012;44(7):1275-86

13. Moher D, Liberati A, Tetzlaff J, Altman DG, PRISMA Group. Preferred reporting items for systematic reviews and meta-analyses: The PRISMA statement. PLoS Med. 2009:6(7):e1000097.

14. Marquet O, Hipp AJ. Worksite built environment and objectively measured physical activity while at work: an analysis using perceived and objective walkability and greenness. J Environ Health. 2019;81(7):20-6.
15. Li Y, Yatsuya H, Hanibuchi T, Hirakawa Y, Ota A, Uemura M, et al. The association between objective measures of residence and worksite neighborhood environment, and self-reported leisure-time physical activities: the Aichi Workers' cohort study. Prev Med Rep. 2018;11:282-9.

16. Prodaniuk TR, Plotnikoff RC, Spence JC, Wilson PM. The influence of selfefficacy and outcome expectations on the relationship between perceived environment and physical activity in the workplace. Int J Behav Nutr Phys Act. 2004;1(1):7.

17. Lucove JC, Huston SL, Evenson KR. Workers' perceptions about worksite policies and environments and their association with leisure-time physical activity. Am J Health Promot. 2007;21(3):196-200.

18. Badland HM, Schofield GM, Garrett N. Travel behavior and objectively measured urban design variables: associations for adults traveling to work. Health Place. 2008;14(1):85-95.

19. de Geus B, De Bourdeaudhuij I, Jannes C, Meeusen R. Psychosocial and environmental factors associated with cycling for transport among a working population. Health Educ Res. 2008;23(4):697-708.

20. Merom D, Miller YD, van der Ploeg HP, Bauman A. Predictors of initiating and maintaining active commuting to work using transport and public health perspectives in Australia. Prev Med. 2008;47(3):342-6.

21. Schwartz MA, Aytur SA, Evenson KR, Rodriguez DA. Are perceptions about worksite neighborhoods and policies associated with walking? Am J Health Promot. 2009;24(2):146-51.

22. Badland HM, Garrett N, Schofield GM. How does car parking availability and public transport accessibility influence work-related travel behaviors? Sustainability. 2010;2(2):576-90.

23. Troped PJ, Wilson JS, Matthews CE, Cromley EK, Melly SJ. The built environment and location-based physical activity. Am J Prev Med. 2010; 38(4):429-38.

24. Wen LM, Kite J, Rissel C. Is there a role for workplaces in reducing employees' driving to work? Findings from a cross-sectional survey from inner-West Sydney, Australia. BMC Public Health. 2010;10:50.

25. Handy SL, Xing Y. Factors correlated with bicycle commuting: a study in six small US cities. Int J Sustain Transp. 2011;5(2):91-110.

26. Panter J, Griffin S, Jones A, Mackett R, Ogilvie D. Correlates of time spent walking and cycling to and from work: baseline results from the commuting and health in Cambridge study. Int J Behav Nutr Phys Act. 2011:8:124.

27. Panter J, Jones AP, van Sluijs EM, Griffin SJ, Wareham NJ. Environmental and psychological correlates of older adult's active commuting. Med Sci Sports Exerc. 2011;43(7):1235-43.

28. Umstattd MR, Baller SL, Blunt GH, Darst ML. Correlates of perceived worksite environmental support for physical activity. J Phys Act Health. 2011;8(s2): S222-S7.

29. Bopp M, Kaczynski AT, Besenyi G. Active commuting influences among adults. Prev Med. 2012;54(3-4):237-41.

30. Buehler R. Determinants of bicycle commuting in the Washington, DC region: the role of bicycle parking, cyclist showers, and free car parking at work. Transport Res Part D-Transport Environ. 2012:17(7):525-31.

31. Bopp M, Kaczynski AT, Campbell ME. Social ecological influences on work related active commuting among adults. Am J Health Behav. 2013;37(4): 543-54

32. Dalton AM, Jones AP, Panter JR, Ogilvie D. Neighbourhood, route and workplace-related environmental characteristics predict adults' mode of travel to work. PLoS One. 2013:8(6):e67575.

33. Heinen E, Maat $K$, van Wee B. The effect of work-related factors on the bicycle commute mode choice in the Netherlands. Transportation. 2013; 40(1):23-43

34. Panter J, Desousa C, Ogilvie D. Incorporating walking or cycling into car journeys to and from work: the role of individual, workplace and environmental characteristics. Prev Med. 2013;56(3-4):211-7.

35. Panter J, Griffin S, Dalton AM, Ogilvie D. Patterns and predictors of changes in active commuting over 12 months. Prev Med. 2013:57(6):776-84.

36. Watts AW, Masse LC. Is access to workplace amenities associated with leisure-time physical activity among Canadian adults? Can J Public Health. 2013:104(1):e87-91.

37. Almeida FA, Wall SS, You W, Harden SM, Hill JL, Krippendorf BE, et al. The association between worksite physical environment and employee nutrition, and physical activity behavior and weight status. J Occup Environ Med. 2014:56(7):779-84. 
38. Bopp M, Child S, Campbell M. Factors associated with active commuting to work among women. Women Health. 2014;54(3):212-31.

39. Bopp M, Der Ananian C, Campbell ME. Differences in active commuting among younger and older adults. J Aging Phys Act. 2014;22(2):199-211.

40. Forsyth A, Oakes JM. Workplace neighborhoods, walking, physical activity, weight status, and perceived health assessing the built environment. Transp Res Record. 2014;2452:98-104.

41. Hamre A, Buehler R. Commuter mode choice and free car parking, public transportation benefits, showers/lockers, and bike parking at work: evidence from the Washington, DC region. J Publ Transp. 2014;17(2):67-91.

42. Karusisi N, Thomas F, Meline J, Brondeel R, Chaix B. Environmental conditions around itineraries to destinations as correlates of walking for transportation among adults: the RECORD cohort study. PLoS One. 2014; 9(5):e88929

43. Panter J, Griffin S, Ogilvie D. Active commuting and perceptions of the route environment: a longitudinal analysis. Prev Med. 2014;67:134-40.

44. Adlakha D, Hipp AJ, Marx C, Yang L, Tabak R, Dodson EA, et al. Home and workplace built environment supports for physical activity. Am J Prev Med. 2015;48(1):104-7.

45. Barrington WE, Beresford SA, Koepsell TD, Duncan GE, Moudon AV. Worksite neighborhood and obesogenic behaviors: findings among employees in the promoting activity and changes in eating (PACE) trial. Am J Prev Med. 2015:48(1):31-41.

46. Piatkowski DP, Marshall WE. Not all prospective bicyclists are created equal: the role of attitudes, socio-demographics, and the built environment in bicycle commuting. Travel Behav Soc. 2015;2(3):166-73.

47. Schoner JE, Cao J, Levinson DM. Catalysts and magnets: built environment and bicycle commuting. J Transp Geogr. 2015;47:100-8.

48. Yang L, Hipp JA, Adlakha D, Marx CM, Tabak RG, Brownson RC. Choice of commuting mode among employees: do home neighborhood environment, worksite neighborhood environment, and worksite policy and supports matter? J Transp Health. 2015;2(2):212-8.

49. Adams EJ, Bull FC, Foster CE. Are perceptions of the environment in the workplace 'neighbourhood' associated with commuter walking? J Transp Health. 2016;3(4):479-84.

50. Bjorkelund OA, Degerud H, Bere E. Socio-demographic, personal, environmental and behavioral correlates of different modes of transportation to work among Norwegian parents. Arch Public Health. 2016;74:43.

51. Clark B, Chatterjee K, Melia S. Changes to commute mode: the role of life events, spatial context and environmental attitude. Transp Res Pt A-Policy Pract. 2016;89:89-105.

52. Mackenbach JD, Randal E, Zhao PJ, Howden-Chapman P. The influence of urban land-use and public transport facilities on active commuting in Wellington, New Zealand: active transport forecasting using the WILUTE model. Sustainability. 2016;8(3):242.

53. Rafferty D, Dolan C, Granat M. Attending a workplace: its contribution to volume and intensity of physical activity. Physiol Meas. 2016;37(12):2144-53.

54. Watts AW, Laska MN, Larson NI, Neumark-Sztainer DR. Millennials at work: workplace environments of young adults and associations with weightrelated health. J Epidemiol Community Health. 2016;70(1):65-71.

55. Adams EJ, Esliger DW, Taylor IM, Sherar LB. Individual, employment and psychosocial factors influencing walking to work: implications for intervention design. PLoS One. 2017;12(2):e0171374.

56. Christiansen P, Engebretsen O, Fearnley N, Hanssen JU. Parking facilities and the built environment: impacts on travel behaviour. Transp Res Pt A-Policy Pract. 2017;95:198-206

57. Gehrke SR, Welch TF. The built environment determinants of activity participation and walking near the workplace. Transportation. 2017:44(5): 941-56.

58. Quinn TD, Jakicic JM, Fertman Cl, Barone GB. Demographic factors, workplace factors and active transportation use in the USA: a secondary analysis of 2009 NHTS data. J Epidemiol Community Health. 2017;71(5):480-6.

59. Yang L, Griffin S, Khaw KT, Wareham N, Panter J. Longitudinal associations between built environment characteristics and changes in active commuting. BMC Public Health. 2017;17(1):458.

60. Batista Ferrer H, Cooper A, Audrey S. Associations of mode of travel to work with physical activity, and individual, interpersonal, organisational, and environmental characteristics. J Transp Health. 2018;9:45-55.

61. Biswas A, Smith PM, Gignac MAM. Naturally occurring workplace facilities to increase the leisure time physical activity of workers: a propensity-score weighted population study. Prev Med Rep. 2018;10:263-70.
62. Carlson JA, Frank LD, Ulmer J, Conway TL, Saelens BE, Cain KL, et al. Work and home neighborhood design and physical activity. Am J Health Promot. 2018;32(8):1723-9.

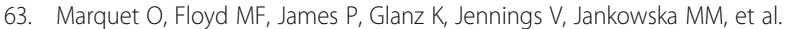
Associations between worksite walkability, greenness, and physical activity around work. Environ Behav. 2018;52(2):139-63.

64. Macdonald L. Associations between spatial access to physical activity facilities and frequency of physical activity; how do home and workplace neighbourhoods in west Central Scotland compare? Int J Health Geogr. 2019;18(1):2.

65. Paul DR, Deng Y, Cook PS. Cross-sectional and longitudinal analysis of the active commuting behaviors of U.S. Department of the Interior employees. BMC Public Health. 2019;19(1):526.

66. Pritchard $R$, Froyen $Y$. Location, location, relocation: how the relocation of offices from suburbs to the inner city impacts commuting on foot and by bike. Eur Transp Res Rev. 2019;11(1):14.

67. Zhang CQ, Zhang R, Gan Y, Li D, Rhodes RE. Predicting transport-related cycling in Chinese employees using an integration of perceived physical environment and social cognitive factors. Transp Res Pt F-Traffic Psychol Behav. 2019;64:424-39.

68. Zhang R, Zhang CQ, Gan Y, Li D, Rhodes RE. Predicting transport-related walking in Chinese employees by integrating worksite neighbourhood walkability and social cognition. Appl Psychol Health Well Being. 2019;11(3): 484-98.

69. McCormack GR, Shiell A. In search of causality: a systematic review of the relationship between the built environment and physical activity among adults. Int J Behav Nutr Phys Act. 2011:8(1):125.

70. National Institutes of Health. Quality assessment tool for observational cohort and cross-sectional studies. 2019 https://www.nhlbi.nih.gov/healthtopics/study-quality-assessment-tools. Accessed 29 Oct 2019.

71. Bort-Roig J, Martin M, Puig-Ribera A, González-Suárez AM, Martínez-Lemos I, Martori JC, et al. Uptake and factors that influence the use of 'sit less, move more' occupational intervention strategies in Spanish office employees. Int J Behav Nutr Phys Act. 2014;11:152.

72. Karmeniemi M, Lankila T, Ikaheimo T, Koivumaa-Honkanen $H$, Korpelainen $R$. The built environment as a determinant of physical activity: a systematic review of longitudinal studies and natural experiments. Ann Behav Med. 2018;52(3):239-51

73. Gebel K, Bauman A, Owen N. Correlates of non-concordance between perceived and objective measures of walkability. Ann Behav Med. 2009; 37(2):228-38

74. Church TS, Thomas DM, Tudor-Locke C, Katzmarzyk PT, Earnest CP, Rodarte $R Q$, et al. Trends over 5 decades in U.S. occupation-related physical activity and their associations with obesity. PLoS One. 2011;6(5):e19657.

75. Chaix B, Simon C, Charreire H, Thomas F, Kestens Y, Karusisi N, et al. The environmental correlates of overall and neighborhood based recreational walking (a cross-sectional analysis of the RECORD study). Int J Behav Nutr Phys Act. 2014;11(1):20.

76. Sugiyama T, Gunn LD, Christian H, Francis J, Foster S, Hooper P, et al. Quality of public open spaces and recreational walking. Am J Public Health. 2015;105(12):2490-5.

77. Bennie JA, Timperio A, Dunstan DW, Crawford D, Salmon J. Environmental correlates of physical activity in Australian workplaces. Int J Workplace Health Manag. 2010;3(1):25-33.

78. Gay JL, Buchner DM, Smith J, He C. An examination of compensation effects in accelerometer-measured occupational and non-occupational physical activity. Prev Med Rep. 2017:8:55-9.

79. Puybaraud M, Russel S, McEwan AM, Luessink E, Beck L. Generation Y and the workplace annual report 2010. London: Intellectual Property Johnson Controls, Haworth and iDEA; 2010.

80. de Sa TH, de Rezende LFM, Borges MC, Nakamura PM, Anapolsky S, Parra D, et al. Prevalence of active transportation among adults in Latin America and the Caribbean: a systematic review of population-based studies. Rev Panam Salud Publica. 2017:41:e35.

\section{Publisher's Note}

Springer Nature remains neutral with regard to jurisdictional claims in published maps and institutional affiliations. 